\title{
Retrofitting Parallelism onto OCaml
}

\author{
KC SIVARAMAKRISHNAN, IIT Madras, India \\ STEPHEN DOLAN, OCaml Labs, UK \\ LEO WHITE, Jane Street, UK \\ SADIQ JAFFER, Opsian, UK and OCaml Labs, UK \\ TOM KELLY, OCaml Labs, UK \\ ANMOL SAHOO, IIT Madras, India \\ SUDHA PARIMALA, IIT Madras, India \\ ATUL DHIMAN, IIT Madras, India \\ ANIL MADHAVAPEDDY, University of Cambridge Computer Laboratory, UK and OCaml Labs, UK
}

OCaml is an industrial-strength, multi-paradigm programming language, widely used in industry and academia. OCaml is also one of the few modern managed system programming languages to lack support for shared memory parallel programming. This paper describes the design, a full-fledged implementation and evaluation of a mostly-concurrent garbage collector (GC) for the multicore extension of the OCaml programming language. Given that we propose to add parallelism to a widely used programming language with millions of lines of existing code, we face the challenge of maintaining backwards compatibility-not just in terms of the language features but also the performance of single-threaded code running with the new GC. To this end, the paper presents a series of novel techniques and demonstrates that the new GC strikes a balance between performance and feature backwards compatibility for sequential programs and scales admirably on modern multicore processors.

CCS Concepts: - Software and its engineering $\rightarrow$ Garbage collection; Parallel programming languages.

Additional Key Words and Phrases: concurrent garbage collection, backwards compatibility

\section{ACM Reference Format:}

KC Sivaramakrishnan, Stephen Dolan, Leo White, Sadiq Jaffer, Tom Kelly, Anmol Sahoo, Sudha Parimala, Atul Dhiman, and Anil Madhavapeddy. 2020. Retrofitting Parallelism onto OCaml. Proc. ACM Program. Lang. 4 , ICFP, Article 113 (August 2020), 30 pages. https://doi.org/10.1145/3408995

\section{INTRODUCTION}

Support for shared-memory parallelism is quite standard in managed system programming languages nowadays. Languages and runtimes such as Go, Haskell, Java, the .NET CLR platform and various parallel extensions of the ML programming language [Fluet et al. 2010; Sivaramakrishnan et al. 2014; Ueno and Ohori 2016; Westrick et al. 2019] all support multiple parallel threads of execution. There has been extensive research and development into efficient garbage collector (GC)

Authors' addresses: K.C. Sivaramakrishnan, A. Sahoo, S. Parimala, A. Dhiman, Department of Computer Science and Engineering, Indian Institute of Technology, Madras, Chennai, India; S. Dolan, T. Kelly, OCaml Labs, Cambridge, UK; L. White, Jane Street, London, UK; S. Jaffer, Opsian and OCaml Labs, Cambridge, UK; A. Madhavapeddy, University of Cambridge Computer Laboratory and OCaml Labs, Cambridge, UK.

This work is licensed under a Creative Commons Attribution 4.0 International License.

(C) 2020 Copyright held by the owner/author(s).

2475-1421/2020/8-ART113

https://doi.org/10.1145/3408995

Proc. ACM Program. Lang., Vol. 4, No. ICFP, Article 113. Publication date: August 2020. 
designs for these languages and runtimes. Our challenge is to retrofit parallelism to the OCaml programming language, which has been in continuous use since 1996 in large codebases, particularly in verification tools or mission-critical systems components. Adding shared-memory parallelism to an existing language presents an interesting set of challenges. As well as the difficulties of memory management in a parallel setting, we must maintain as much backwards compatibility as practicable. This includes not just compatibility of the language semantics, but also of the performance profile, memory usage and $\mathrm{C}$ bindings.

\subsection{Performance Backwards Compatibility}

The main challenge in adding support for shared-memory parallelism to OCaml is the implementation of the multicore-capable GC. OCaml users have come to rely on allocation and memory access being relatively cheap (for a managed language) in order to write performant code. Being a functional programming language, OCaml code usually exhibits a high rate of allocation with most objects being small and short-lived. Hence, OCaml uses a small minor heap with a generational GC to collect the small, short-lived objects. Objects are allocated in the minor heap by bumping the allocation pointer. Hence, the minor heap allocations are very fast.

Many objects in OCaml are immutable. For immutable objects, the initialising writes (the only ones) are done without barriers and reads require no barriers. For mutable fields, the writes require a (deletion/Yuasa/snapshot-at-the-beginning [Yuasa 1990]) barrier, and the reads do not. Hence, reads are fast and updates are comparatively slower.

Objects that survive a minor collection are promoted to the major heap, which is collected by an incremental, non-moving, mark-and-sweep collector, with an optional compaction phase. This design minimizes pause times, and indeed, OCaml is used to write latency sensitive applications such as network services (MirageOS [Madhavapeddy et al. 2013]) and user interfaces (ReasonML [ReasonML 2019]). Our aim with the multicore capable GC is to preserve the fast performance characteristics of operations as they currently exist on single-core OCaml, with some compromises for mutable objects to deal with the possibility of racy access between multiple mutator and GC threads. We strive to retain the low pause times of single-core OCaml.

An alternative way to achieve performance backwards compatibility for those programs that do not utilise parallelism would be to have two separate compilers and runtimes for the serial and parallel code. For example, GHC Haskell comes with two separate runtimes, one which supports parallelism and another which is purely single-threaded [Marlow et al. 2009]. GHC provides a compiler flag - threaded to choose the runtime system to link to. In order to avoid the maintenance burden of two separate runtimes, we chose to go for a unified runtime.

\subsection{Feature Backwards Compatibility}

Given the millions of lines of OCaml code in production, it would serve us well to ensure that the addition of parallelism to OCaml breaks as little code as possible. OCaml is a type-safe language and the addition of parallelism should not break type safety under data races. Dolan et al. present a memory model for shared-memory parallel programs that gives strong guarantees (including type safety) even in the presence of data races [Dolan et al. 2018b]. They describe the instantiation of the memory model for the OCaml programming language and demonstrate that the memory model has reasonable overheads. Our work inherits this memory model.

Beyond type safety, OCaml has several features that closely interact with the garbage collector. These include weak references, finalisers, ephemerons [Hayes 1997], and lazy values, whose semantics will have to be preserved with the new GC so as to not break programs that use those features. OCaml's C API exposes quite a lot of the internals of the memory representation, and has an expressive API to access the heap efficiently [OCamlCAPI 2019]. While this allows users to

Proc. ACM Program. Lang., Vol. 4, No. ICFP, Article 113. Publication date: August 2020. 
write fast code, the API also bakes in the invariants of the GC. For example, reading any OCaml object, be it mutable or not, using the C API does not involve a read barrier, and is compiled as a plain read of memory. A new GC scheme that adds a read barrier only to reads of mutable fields will need to deprecate the old API or suffer the risk of breaking code silently. Either way, the users will have to modify their code to work correctly under the new GC. Given that the compiler does not check incorrect uses of the C API, it is already difficult to write correct and efficient FFI code. We would like to strike a balance between the added complexity of the C API and the performance impact of the missed opportunities.

\subsection{Requirements}

In summary, we strive towards the following ideal goals in our parallel extension of OCaml:

R1 A well behaved serial program does not break on the parallel extension. That is, a well-typed serial program remains well-typed in the parallel extension, and the semantics of such a program remains the same on the serial and parallel runtimes.

R2 The performance profile of a serial program on the parallel runtime remains the same as the serial runtime. That is, the program on the parallel runtime should run as fast as it does on serial runtime. Additionally, the GC pause times of the serial program on the parallel runtime remain the same as the serial runtime.

R3 The parallel programs should aim to minimize pause times, and then aim to run as fast as possible on the available cores. We order the sub goals this way since minimising pause times in the GC is much harder than achieving good throughput. We believe that once the pause times are optimised for, optimising for throughput is easier, but the vice versa is much harder.

We develop a generational garbage collector with two generations where the old generation (major heap) is shared between all of the mutators and is collected with a non-moving, mostlyconcurrent, mark-and-sweep collector modelled on VCGC [Huelsbergen and Winterbottom 1998]. VCGC avoids having explicit phase transitions between marking and sweeping, which is the traditional source of bugs in concurrent collector designs. For programs that do not use weak references or ephemerons, the mutator and the GC threads need to synchronize only once per cycle to agree that the current cycle is done. This minimizes the pause times. The major heap allocator is based on the Streamflow [Schneider et al. 2006] design, which uses size-segmented thread-local pages. This has been shown to have good multicore behaviour and fragmentation performance.

For the young generation (minor heap), survivors are copied to the shared major heap. We present two alternative collectors for the minor heap with different tradeoffs (§4). The first is a concurrent collector with thread-private minor heaps [Anderson 2010; Auhagen et al. 2011; Doligez and Leroy 1993; Domani et al. 2002; Marlow and Peyton Jones 2011; Sivaramakrishnan et al. 2014]. The original quasi-real-time collector design by [Doligez and Leroy 1993] maintains the invariant that there are no pointers from major to minor heaps. Thus, storing a pointer to a private object into the shared major heap causes the private object and all objects reachable from it to be promoted to the shared heap en masse. Unfortunately, this eagerly promotes many objects that were never really shared: just because an object is pointed to by a shared object does not mean another thread is actually going to attempt to access it. A good example is a shared work-stealing queue, where stealing is a rare operation. It would be unwise to promote all the work to the major heap.

Our concurrent minor collector design $(\S 4.3)$ is similar but lazier, along the lines of GHC's local heaps [Marlow and Peyton Jones 2011], where objects are promoted to the shared heap whenever another thread actually tries to access them. This has a slower sharing operation, since it requires synchronisation of two different threads, but it is performed less often. However, this design requires that reads be safe points where garbage collection can occur. Consider the case of two 
mutators both of which are trying to read an object in the minor heap of the other mutator. In order to make progress, the mutators will have to service promotion requests that they receive while waiting for their request to be satisfied. Recall that stock OCaml does not use read barriers and the C API also works under this assumption. By making the reads safe points, it is likely that every user of the C API will need to update their code to conform with the new semantics. This conflicts with our first requirement.

To this end, we develop a stop-the-world parallel minor collector $(\S 4.4)$ where all the mutators will need to synchronize to perform the minor collection, at the cost of possible worse pause times. The memory access invariants remains the same, and hence, no change is necessary for the C API. One surprising result we will justify in our evaluation (§6) is that the stop-the-world minor collector outperforms the concurrent minor collector in almost all circumstances, even as we cranked up the number of cores. This result gives us a clear choice in the design space to pick a backwards-compatible and performant concurrent garbage collector.

\subsection{Contributions}

Our contributions are to present:

- the design of a mostly-concurrent, non-moving, mark-and-sweep GC for the older generation that minimizes pause times for a parallel extension of OCaml.

- two collector designs for the young generation: (i) a concurrent collector that minimizes pause times at the cost of breaking the C API and; (ii) a stop-the-world parallel collector that retains the backwards compatibility of the existing C API.

- extensions of our baseline collectors to advanced language features that interact with the GC such as lazy values, finalisers, weak references and ephemerons. Our novel design minimizes the number of global synchronizations necessary for collecting a deeply nested hierarchy of ephemerons. This design has been verified in the SPIN model checker.

- support for fibers that run in parallel, which are language level lightweight threads implemented as runtime managed stack segments. The implementation of fibers is similar to lightweight threads in Haskell GHC and Goroutines in the Go language. While the details of the language support for fibers is beyond the scope of the paper, we describe the subtle interaction of our concurrent GC algorithm with fibers.

- extensive evaluation of the collector designs in a full-fledged implementation of a parallel extension of OCaml. Our experiments illustrate that $(i)$ serial programs retain their performance profile on the new collectors, and (ii) parallel programs achieve good multicore scalability while preserving low pause times with increasing number of cores.

The rest of the paper is organized as follows. In the next section, we give an overview of memory management and garbage collection in OCaml, which applies to both stock OCaml and our parallel extension. §3 gives a detailed description of the major heap allocator and collector, and the changes required to make it parallel, while $\S 4$ does the same for the minor heap. $\S 5$ describes the extension of the new GC with lazy values, finalisers, weak references, ephemerons, and fibers. §6 presents extensive performance evaluation of the new GC against the goals set in §1.3. In the light of this performance evaluation, $\S 7$ discusses the path ahead for retrofitting parallelism onto OCaml. §8 and $\S 9$ present the related work and conclusions.

In the remainder of the paper, we call our parallel extension of OCaml "Multicore OCaml" to distinguish it from stock OCaml. Following standard GC terminology, we refer to the user program as "the mutator", even though actual mutation is relatively rare in OCaml programs. 


\section{AN OVERVIEW OF MEMORY MANAGEMENT IN OCAML}

OCaml uses a uniform memory representation in which each value has the same size [Leroy 1990], making it possible to compile just one copy of polymorphic functions [Appel 1990]. Values are always one word long (either 32 or 64 bits, depending on architecture), and consist of either an integer or a pointer. The least significant bit (LSB) is used to distinguish integers and pointers: since all allocations are word-aligned, the LSB of pointers is guaranteed to be zero, whereas integers are encoded by left-shifting the integer value by one, and setting the LSB to one. Hence, integers in OCaml are 31- or 63-bits long. Every OCaml object has a word-sized header, which encodes the length and type of the object, and has two bits reserved for encoding the colours used by the major collector. (For more details of OCaml's memory representation, see Minsky et al. [2013, Ch. 20]).

OCaml uses a generational GC with a major and minor heap [Doligez 1989]. The minor heap is small ( $256 \mathrm{~K}$ words, by default), where new objects are allocated by bumping the allocation pointer. The minor heap is collected when it is full by a copying collector which copies live objects to the major heap. The minor collection proceeds by first promoting all the roots (globals, local roots registered in $\mathrm{C}$ calls, registers, the remembered set of inter-generational pointers from the major to the minor heap, and the program stack) that point to the minor heap to the major heap, and then transitively promoting all the referenced objects. All references from live objects to objects in the minor heap are updated to point at the new location, including references from the major heap to the minor heap (recorded in the remembered set), and the old minor heap is then reused in the next cycle. The copying collector only needs to touch the live objects. Given that the survival rate in the minor collection is low, a copying collector minimizes pause times.

The major heap contains objects which have survived a minor collection (as well as objects above a certain size, which are allocated there directly). Instead of a bump-pointer algorithm, allocation in the major heap uses a more sophisticated allocator, which differs between stock and Multicore OCaml. The major GC is incremental, except for an optional stop-the-world compaction phase.

\section{MAJOR HEAP}

Next, we present the details of OCaml's major heap and garbage collector, and the changes necessary to retrofit parallelism. OCaml's major collector is:

Mark-and-sweep Collection is divided into two phases: marking determines which allocations are still in use, and sweeping collects those that are not and makes them available for reuse.

Non-moving Once allocated in the major heap, objects remain at the same address until collected. Incremental Rather than stopping the program for the whole duration of GC (which would cause a pause bounded in length only by the size of memory), the OCaml major collector pauses the program in many small slices.

Multicore OCaml's collector retains these properties, and is also parallel: the virtual machine contains a number of domains, each running as a separate system thread in the same address space. Domains can be dynamically created and brought down.

A garbage collector uses a large amount of shared mutable state, as the collector must track how each byte of memory is being used. The central challenge in writing a multicore garbage collector is controlling access to this state without introducing too much expensive synchronisation. To understand this challenge, we first review how this state is used in the stock OCaml collector.

\subsection{Tricolour Collection in Stock OCaml}

OCaml uses the standard tri-colour abstraction [Dijkstra et al. 1978]: allocations on the major heap are given one of three colours black, gray and white. The colour of an object is stored in the two 
GC bits in the object's header. The fourth possible value of these bits, named blue by OCaml, is used to indicate blocks that are not objects but free memory, available for future allocations.

The collector operates in two phases, first marking and then sweeping. These phases are incremental and so are interleaved with the user program (Figure 1), which in particular may mutate the heap and allocate new objects.

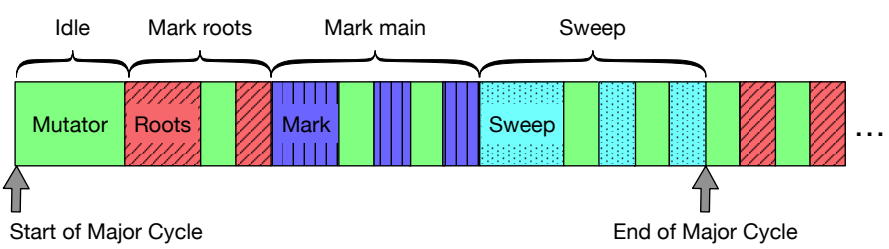

Marking. The goal of the

Fig. 1. Major collection in stock OCaml marking phase is to colour all live objects black, leaving dead (garbage) objects coloured white. The gray objects represent the frontier, that is, those objects which have themselves been marked but which have not yet been scanned for outgoing pointers.

The first part of marking is to mark the roots. Marking makes a white object grey and pushes it into the mark stack. The root marking is itself split into two phases - a non-incremental phase where the registers and the program stack are scanned, and an incremental phase for scanning the global roots. Given that real world programs may have a large number of global roots, incrementally marking them reduces pause times.

After the roots are marked, marking is continued by popping the mark stack, marking the children of the object popped, and then marking the object black. This process is repeated until the mark stack becomes empty, meaning that there are no more gray objects.

Sweeping. When marking is completed, the objects that are still in use are black and the garbage is white. The sweeping pass does a single traversal of the whole heap (again, incrementally), turning black objects back to white and turning white objects blue, to indicate that their storage is now free to be used for future allocations.

Mutation. When mutating a field of an object, the program invokes the write barrier. During the mark phase of the GC, the write barrier loads the object pointed to by the old value of the field, and if it is white, grays it and adds it to the mark stack. This preserves the invariant that every object reachable at the start of marking eventually gets marked (the snapshot-at-the-beginning property)

The write barrier also keeps track of the inter-generational pointers from major to minor heap in a remembered set, which is used as a root for the minor collection. (See section 4).

Allocation. New allocations must be coloured so that they are not immediately collected. During the marking phase, this means that they are coloured black. During the sweeping phase, their allocation colour depends on whether sweeping has yet reached their position: if so, they are coloured white, but if not, they are coloured black so that the sweeper later turns them white.

This is just one possibility from a large design space. OCaml grays the old value of a field during mutation (a deletion barrier [Yuasa 1990]) and marks the roots before marking anything else (a black mutator). This has the advantage that the total amount of work to do during a GC cycle is bounded, regardless of what the mutator does (guaranteeing termination of GC), but the disadvantage that anything allocated on the major heap during the marking phase of one cycle can be collected at the earliest a full cycle later. See Vechev et al. [2005] or Jones et al. [2011] for further discussion of these details. We will not discuss them further here, as the trade-offs involved are largely independent of the switch from a single-threaded to a multi-threaded GC. 
What we do discuss here is the shared state involved in this design. Mutable state is shared between marking and sweeping (the object colours, whose meaning changes between phases), between mutation and marking (the write barrier supplies new gray objects to the collector) and between allocation and sweeping (allocation must determine the phase of the collector and position of sweeping, as well as coordinating with the sweeper to manage free memory).

In a single-threaded collector, organising access to such shared state is easy, but doing so correctly and efficiently is the central challenge of a parallel collector. Even the seemingly-simple state of whether the collector is marking or sweeping is problematic: many designs have had errors in the case where the GC transitions phase during a write barrier, relying on the precise order of operations [Gries 1977], which becomes even trickier under weak memory [Gammie et al. 2015].

\subsection{Multicore OCaml's Collector}

The approach we take in multicore OCaml is to avoid as much as possible of this shared state. First, to reduce state shared between mutators and GC, we do not use an explicit gray colour. Instead, each domain maintains its own stack of gray objects, and when the write barrier needs to gray an object it marks it and adds it to the local domain's gray stack. Since GC and OCaml code are interleaved on a single domain, no synchronisation is necessary. (This scheme does mean that it's possible for an object to be on the mark stack of two separate domains. See below).

Avoiding shared state between marking and sweeping is trickier, and to this end we reuse a design from the Very Concurrent Garbage Collector (VCGC) [Huelsbergen and Winterbottom 1998], which does not have distinct marking and sweeping phases. Instead of colouring objects black and white (with the meaning of black and white changing as we move between mark and sweep phases),

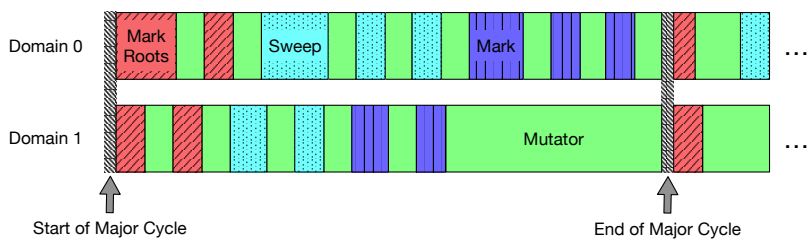

Fig. 2. Major collection in Multicore OCaml. Our design permits the marking and sweeping phase to overlap and avoids the global synchronization between the phases.

Multicore OCaml uses three states:

Marked, Unmarked and Garbage. For regions of free memory, there is a fourth state Free, corresponding to OCaml's blue.

Marking proceeds by changing Unmarked objects into Marked ones, while sweeping proceeds by collecting Garbage objects into Free blocks. Note that the sets of objects affected by marking and sweeping are disjoint, so no synchronisation between them is necessary. Likewise, new allocations are always Marked, ensuring that they will not be immediately collected. No synchronisation with the collector is required to determine allocation colour.

As well as state shared between the various phases of the collector and the mutator itself, in a parallel collector we must also think about state shared between multiple domains doing the same phase of the collector in parallel. We resolve this by making marking idempotent and sweeping disjoint. Multiple domains may attempt to mark the same object at the same time, and we make no attempt to avoid this. Instead, we allow the occasional object to be marked twice, knowing that this gives the same result as marking once. Allowing for this possibility is much cheaper than synchronising each access to an object by the collector.

Sweeping is not idempotent. Instead, we ensure that the areas swept by different domains are disjoint: each domain sweeps only the memory that it allocated, keeping the interaction between sweeping and allocation local to a domain. 
Any new domains that are created in a cycle will not have any marking work to do in that cycle. This is because any of the new domain's roots will have to (transitively) come from one of the domains that were running at the end of the previous cycle, and will either have been marked by another domain or were allocated already Marked. Hence, they are not included in gNumDomsToMark.

\subsection{Allocator}

Since all values allocated in OCaml are immediately initialised, a performance goal for the allocator is to make the cost of allocation roughly proportional to the cost of initialisation. In other words, small objects (lists, options, etc.) must be allocated quickly, but larger allocations may be slower.

In stock OCaml, there is a choice of major heap allocators. The first-fit and next-fit policies are classical single-free-list allocators, satisfying allocation requests from the first sufficiently large free block or the next one after the previous allocation, respectively. Recently, a best-fit allocator has been incorporated into OCaml [Doligez 2019] which uses segregated free-lists [Standish 1980] for small object sizes (the common case), and a splay tree [Sleator and Tarjan 1985] of blocks of different sizes for larger allocations. Best-fit has been observed to beat the existing algorithms on both time spent and space used through fragmentation.

However, all of these allocators are single-threaded. Due to the high allocation rates in OCaml, using a lock to protect the allocator would have an unacceptably high performance penalty. For this reason, Multicore uses a different major heap allocator based on the Streamflow [Schneider et al. 2006] design. Our allocator maintains a domain-local, size-segmented list of pages for small allocations (less than 128 words). Each page of $4 \mathrm{~K}$ words is carved into equal-sized slots, with size classes chosen so that there is less than $10 \%$ wasted space.

Large allocations (at least 128 words) are forwarded to the system malloc, maintained in a domainlocal list of large blocks, and returned via system free when swept. Before a domain terminates, all of the pages it owns are moved to a global size-segmented list of pages. Such pages are said to be orphaned and must be serviced by the other domains as they might contain live objects referenced by other domains. The access to this global list of pages is protected by a mutex.

Streamflow uses BiBoP [Steele Jr 1977] to track which slots in a page are free without requiring object headers. However, since every OCaml object already has a header, we use it instead to encode which slots are free.

In order to service an allocation request to the major heap, the domain searches for a suitable slot in the corresponding size class in its local list of pages. If no such slot is found, the domain sweeps the local unswept pages of that size class to find a free slot. Failing that, the domain adopts one of the global pages of the appropriate size class with an available slot. Failing that, the domain adopts one of the full global pages of the appropriate size class, sweeps it, and looks for a free slot. If that case fails too, the domain will request the operating system to allocate an additional page. Thus, most allocations can be serviced without the need for synchronization and atomic operations.

\subsection{Safe Points}

In order to support the stop-the-world pauses required by major collector cycle changes and the parallel minor collector, multicore OCaml uses safe points in addition to the existing allocation points. This is implemented using the algorithm from [Feeley 1993] and bounds the number of instructions until a safe point is encountered. These safe points are necessary to avoid deadlocks in certain situations e.g one domain in a non-allocating loop spin-waiting on an empty queue while all other domains need a minor collection in order to allocate new items to put on the queue. It should be noted that safe points are required by stock OCaml for correct language semantics independent of multicore. 


\subsection{Opportunistic Work}

The incremental, concurrent nature of the major collector allows work to be carried out opportunistically when domains might otherwise be idle or in a state where it is unsafe for mutators to be running. Multicore OCaml makes use of this property of the major collector to schedule opportunistic marking and sweeping work in the following cases:

Yielding Many non-blocking algorithms involve some form of looping when attempting to make progress in the face of contention and involve some relaxing e.g PAUSE on x86. We use this as an opportunity to do a small amount of opportunistic marking or sweeping.

Stop-the-world entry waits In the parallel minor collector domains entering a stop-the-world pause will carry out opportunistic work until all domains are ready.

There are additional areas where opportunistic work could be implemented in Multicore OCaml in the future, such as via read faults or stop-the-world leave waits, but these are left as future work.

\section{MINOR HEAP}

The minor heap is much smaller than the major heap, but must sustain a much higher rate of allocation and collection. Unlike the major collector, the minor collector is:

Copying At collection time, live objects in the minor heap are moved to the major heap. The minor heap is emptied by this process, and its space is reused in the next cycle.

Non-incremental The mutator is paused for the entire duration of the minor collection.

As in the previous section, we first review OCaml's single-threaded algorithm.

\subsection{Copying Minor Collection in Stock OCaml}

Like the major collector, the minor collector traces objects from the roots to find the live objects. Unlike the major collector, the set of roots also includes the remembered set, which consists of the references from the major heap to the minor heap. This way, all objects in the minor heap that are pointed to by something in the major heap at the time of minor collection get promoted, without having to traverse the major heap to find them.

The number of objects to be copied by the minor collector is often small. This is for two reasons: first, the minor heap is relatively small, and second, only that part of the minor heap that is live at collection time must be traversed. The limited work needed by the copying collector enables low pause times to be achieved.

Since objects in the minor heap will be discarded or moved soon, there is no need to use a sophisticated allocator to place them. Allocation is therefore fast with a simple bump-pointer allocator.

\subsection{Parallelising the Minor Collector}

Compared to the major collector, the minor collector is more difficult to parallelise as it moves objects, and an object must not be moved by one thread while another is using it.

It is possible to parallelise a copying collector by carefully coordinating the collector and the mutator, sharing state about which objects have been moved. Such an approach comes at a heavy price, however, in either performance or implementation cost, as it requires fine-grained synchronisation between the collector and mutator not just on mutations but on all memory accesses.

So, we avoid designs which involve copying objects in parallel with their use by the mutator. This leaves two possibilities: to separate the minor collector from the mutator in time or in space.

Separation in time When the minor heap fills, stop all domains simultaneously, and have all domains collect the minor heap in parallel before resuming the mutator. 
Separation in space Give each domain a private minor heap, preventing any access by one domain to another's heap, and allow them to collect their minor heaps independently.

We have implemented both of these approaches, as described below and evaluated in section 6 .

\subsection{Concurrent Minor Collector with Private Minor Heaps}

Our concurrent minor collector uses domain-local, private, minor heap arenas, each tracking their own remembered set. We maintain the invariant that there are no pointers between the minor heaps of different domains, which permits each of the minor heaps to be independently collected. We do allow pointers from the shared major heap to the minor heap to avoid paying the cost for early promotion, similarly to multicore GHC [Marlow and Peyton Jones 2011].

4.3.1 Read Faults and Interrupts. Whenever a read follows a pointer from major to a remote minor heap, it triggers a read fault. The faulting domain issues an interrupt to the remote domain to request promotion of the desired object and waits for the response. Each domain maintains a multiple-producer single-consumer queue for receiving inter-domain interrupts. The sender pushes the request into the target domain's queue and modifies the minor heap allocation limit pointer on the target domain such that the next allocation on the target domain would fail. This enables timely handling of inter-domain interrupts. We use the same interrupt mechanism for signalling to other domains when a stop-the-world phase is necessary at the end of the major cycle (§3.2.2).

When the target domain receives a promotion request, it promotes the transitive closure of the requested object to the major heap (possibly triggering a minor GC on this domain), and returns the location of the new object in the major heap to the source domain. Since two domains may concurrently request promoting objects from each other's domains, while the faulting domain waits for a response from the remote domain, it polls its interrupt queue and handles requests.

\subsubsection{Read Barrier. The main challenge in the con-} current GC design is optimising the read barrier, which is a code size and execution time burden not present in stock OCaml.

There is an efficient way to implement the read barrier through careful virtual memory mapping for minor heap arenas and bit twiddling. When reading a value from a mutable location, the read barrier must classify the value as: (a) an integer; or (b) a value in the major heap; or (c) a value in its own minor heap; or $(d)$ a value in a remote minor heap. We must distinguish the last case from the others,

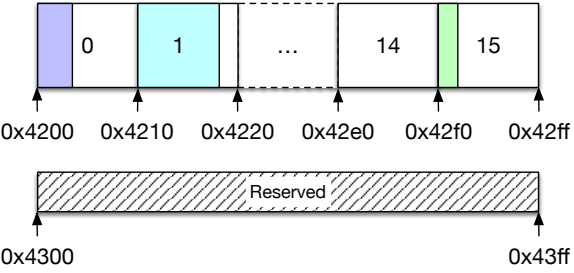

Fig. 4. An example virtual memory mapping of the minor heaps for the concurrent minor collector on a 16-bit address space. as it requires a read fault.

Figure 4 shows an example layout of the minor heap arenas, using only 16-bit addresses for clarity. The minor heaps are all allocated in a contiguous power-of- 2 aligned virtual memory area, where each minor heap is also a power-of- 2 aligned and sized. Not all of the virtual memory area needs to be allocated, but only needs to be reserved to prevent it being used for the major heap. In the figure, the shaded regions indicate committed memory currently used for minor heap arenas.

In this example, the minor heap arenas are all carved out of the virtual memory space between $0 \times 4200$ and $0 \times 42 \mathrm{ff}$. We also reserve the virtual memory space $0 \times 4300$ to $0 \times 43 \mathrm{ff}$, and allocate neither minor or major heap pages in this space. We have chosen a layout with support for 16 domains, each with a maximum minor heap size of 16 bytes. Addresses in this 16-bit address space can be written as 4 quads $0 \times P Q R S$. In OCaml, integer values are represented by tagging the least significant 
113:12 K.C. Sivaramakrishnan, S. Dolan, L. White, S. Jaffer, T. Kelly, A. Sahoo, S. Parimala, A. Dhiman, A. Madhavapeddy

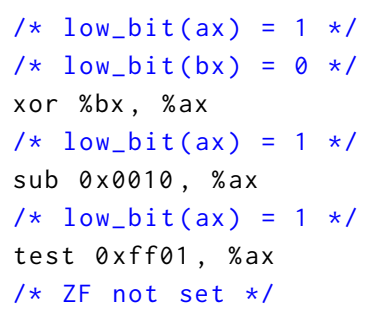

(a) Case: Integer

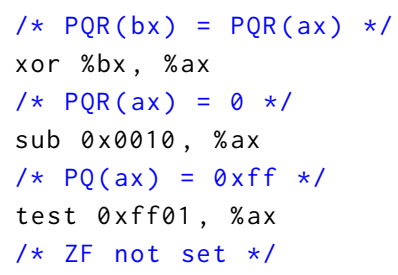

(c) Case: Own minor heap

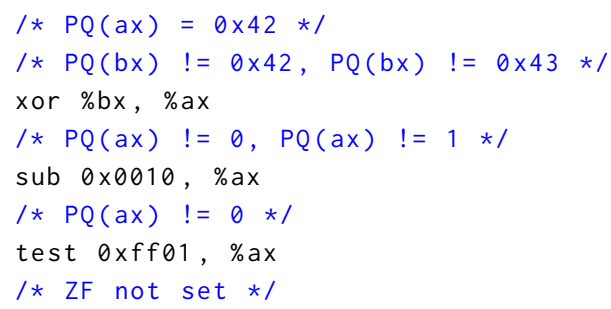

(b) Case: major heap

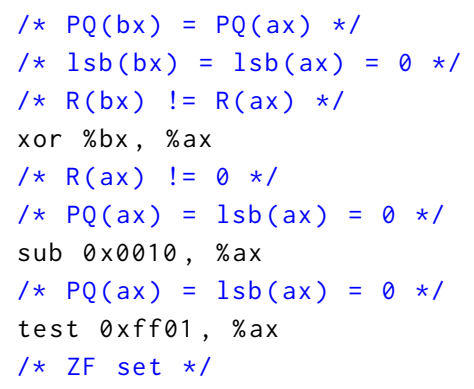

(d) Case: Remote minor heap. 1sb returns least significant bit.

Fig. 6. Proof of correctness of the read barrier.

bit to be 1 . Hence, in this example, integers have low bit of $S$ to be 1 . Minor heap values have PQ to be 42 , and $\mathrm{R}$ determines the domain.

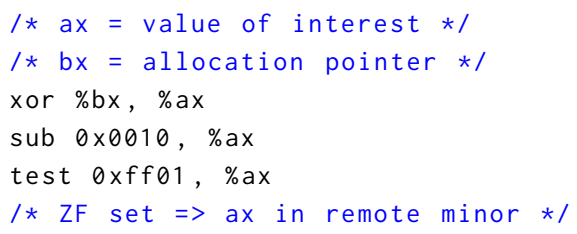

Fig. 5. Read barrier test
We can implement the read barrier check by comparing the given address with an address from the minor heap. Luckily, we have such an address handily available in a register- the minor heap allocation pointer. Let us assume the $\mathrm{x} 86$ architecture, with the allocation pointer in the bx register, and the value that we want to test in ax. The read barrier test is given in Figure 5. The test instruction performs bitwise and on the operands, and sets the zero-flag (ZF) if the result is 0 . At the end of this instruction sequence, if the zero-flag is set, then ax is in a remote minor heap. To see how this works, let us consider each of the four cases (Figure 6).

Consider the case when ax contains an integer (Figure 6a). Since the allocation pointer is always word aligned in OCaml, the least significant bit of the allocation pointer bx will be 0 . Hence, zero flag will not be set at the end of the instruction sequence.

Consider the case when ax contains a major heap pointer (Figure 6b). Due to our virtual memory layout for minor heaps which reserves additional unused virtual memory area adjacent to the used minor heap area, the most significant eight bits in bx (written $P Q(b x)$ ), will neither be $0 \times 42$ nor $0 \times 43$. Hence, $P Q(a x)$ after xor will be non-zero. Hence, zero flag will not be set at the end of the instruction sequence. 
Consider the case when ax contains a pointer to the domain's own minor heap (Figure 6c). In this case, $\mathrm{PQR}(a x)=\mathrm{PQR}(\mathrm{bx})$. After xor, $\mathrm{PQR}(\mathrm{ax})$ is 0 . The subsequent subtraction underflows, and hence, the test does not set the zero flag.

Consider the case when ax contains a pointer to a remote minor heap (Figure 6d). In this case, $P Q(a x)=P Q(b x)$, the least significant bits (lsb) of $a x$ and $b x$ are 0 , and $R(a x) \quad !=R(b x)$. Hence, after xor, $\mathrm{R}(\mathrm{ax}) \quad !=0, \mathrm{PQ}(\mathrm{ax})=1 \mathrm{sb}(\mathrm{ax})=0$. Subtracting 1 from the non-zero $\mathrm{R}(\mathrm{ax})$ will not underflow Hence, $P Q(a x)$ and $l s b(a x)$ are still 0 after subtraction, and the zero flag is set. At this point, the domain raises the interrupt to the target domain to promote the object to the major heap.

Our implementation allows the maximum size of the minor heap and the maximum number of minor heap arenas (and hence, the number of domains) to be configured at the compiler build time. On 64-bit architectures, by default, the compiler is configured to host a maximum of 128 domains, each with a maximum minor heap size of $16 \mathrm{MB}$. This necessitates reserving (not allocating) 4GB of virtual address space, which is a small portion of that available on a 64-bit machine.

4.3.3 Promotion. When a domain receives a promotion request, how should it go about doing it? Because of mutable fields (and finalisers), it is not valid to duplicate the requested object closure in the major heap, and we must ensure all references are updated to the promoted version.

A correct but inefficient implementation would be to perform a minor garbage collection, which will ensure that the requested object and everything else in the minor heap is promoted to the major heap. This strategy suffers from early promotion, and both the requester and the target domain will suffer the pause time for a full minor GC on the target domain.

Observe that we can fix the early promotion problem by promoting only the transitive closure of the requested object, and then scanning the minor GC roots and the minor heap for pointers to the promoted objects and forwarding them to the major heap copy. However, this solution is also inefficient, as it would touch the entire minor heap (recall that the copying collector need only touch live objects during a minor GC, a small portion of the heap).

Our solution is a hybrid of the above techniques. In our experiments, we observe that most promoted objects were recently allocated (as was observed in the case of a parallel extension of the MLton Standard ML compiler [Sivaramakrishnan et al. 2012]). We enhance the write barrier to record in a domain-local minor remembered set the writes from old to younger minor heap objects. How do we identify whether an intra-minor-heap pointer is from an old to a younger object? In OCaml, the allocation pointer starts at the end of the minor heap area, and objects are allocated in the minor heap by subtracting the allocation pointer by the required size. During a write $r:=v$, if both $r$ and $v$ are objects in the minor heap, and $r$ is at a higher address than $v$, then $r$ is older than $v$. We add $r$ to the minor remembered set.

When an object closure being promoted is recently allocated (in the last $5 \%$ of the minor heap), we promote the object closure. The pointers to those promoted objects may only appear in either the minor GC roots, the minor remembered set, or one of the objects allocated after the oldest promoted object, which is by definition in the last $5 \%$ of the minor heap. We scan those data structures after object promotion. In our experiments, $95 \%$ of the promoted objects were the youngest $5 \%$ of objects. If the object closure being promoted is not one of the youngest $5 \%$, we perform a full minor collection. The minor remembered set is cleared at the end of a minor collection.

4.3.4 Rewriting Remembered Set. In Multicore OCaml, the domain-local remembered set records the pointers from the shared major heap to the minor heap, which must be updated during minor collection. Consider that a major heap object $r$ points to the minor heap object $\mathrm{m}$. After promoting $\mathrm{m}$, $r$ should be rewritten to point to the new location of $m$ in the major heap, say $m$ '. However, care must be taken to ensure that this write by the minor GC does not overwrite a concurrent write to $r$ by a different domain. To this end, we perform the write to $r$ by the minor GC with an unconditional 
atomic compare-and-swap CAS $\left(r, m, m^{\prime}\right)$, which will fail if $r$ does not contain $m$. If it fails, then $r$ has been written to by a different domain, and hence, we ignore the failure.

\subsection{Stop-the-world Parallel Minor Collector}

In this section, we describe the stop-the-world parallel minor collector which is an alternative to the concurrent minor collector. Our stop-the-world parallel minor collector retains domain-local minor heaps but relaxes the invariant disallowing references between minor heaps. Inter-heap references requires a stop-the-world synchronisation involving all domains to carry out a minor heap collection. With stop-the-world minor collection, we do not need read barriers and the read barriers need not be safe points. Hence, we can retain the stock OCaml's C API.

4.4.1 Interrupts and Synchronization. When a domain finds that it needs to collect its minor heap, it utilizes the interrupt mechanism to signal that all domains should do a stop-the-world collection. Care is taken to ensure that stop-the-world minor and major collection requests are serialized so that only one can be executing at a given time.

Given the increase in the frequency of interrupts, safe points become necessary for the stopthe-world minor collector to avoid long synchronization times before a minor collection can start. When all domains have entered the interrupt a barrier is used to agree that a minor collection can start; at this point we know that all the blocks in the minor heap can be safely moved.

4.4.2 Parallel Promotion. Within the stop-the-world section, all domains promote reachable objects in parallel. Care is needed to ensure that if two domains attempt to promote an object, they are serialized using CAS operations on the object header. This is a little more involved than a single CAS due to the need to update the object's first field to forward to the new location in the major heap. Other domains must never see a minor heap object with a zeroed header (indicating promoted) before the first field is updated. In order to avoid this, the header is CASed to an intermediate state which indicates that a domain is currently promoting the object. If a domain sees this intermediate state when attempting to promote an object they spin until the header is set to $\theta$, at which point the first field now contains the forwarding pointer to the promoted object in the major heap.

Additionally, we incorporate fast paths for the case when there is only one domain running, which elides the CAS and the intermediate states. The minor collection algorithm in the case when only one of the domains is running involves the same steps as stock OCaml minor collection.

4.4.3 Parallel Work Sharing. We implement a static work sharing policy for speeding up the stopthe-world remembered set promotion. This is necessary because each domain maintains its own remembered set and there can be workloads where this leads to imbalance e.g only one domain continually updates a set of globals with newly allocated data. The static work sharing policy equally splits the remembered set entries across all domains participating in the minor collection and aims to ensure each one has an equal number of roots to promote.

Static work sharing only balances the roots in the remembered set. It is entirely possible that one domain may end up needing to promote a large object graph reachable from one of its roots while other domains remain idle. This could be addressed with dynamic work sharing, though it is unclear whether the benefits would outweigh additional synchronisation between domains.

\section{COMPLETING OCAML LANGUAGE COVERAGE}

OCaml has a substantial existing base of production code that has been developed with single-core performance in mind. Some of the popular areas the language is used involve symbolic computation (theorem provers, proof assistants, static analysis, etc.) and these will take some years to migrate to parallel execution. Therefore, our decisions about how to retrofit parallelism have been heavily

Proc. ACM Program. Lang., Vol. 4, No. ICFP, Article 113. Publication date: August 2020. 
influenced by the need to preserve fast single-core performance, as it is impractical to maintain multiple runtimes.

We will now describe the extensions of our GC model needed to handle the complete set of features in OCaml that interface with the GC, paying careful attention to single-core performance to minimise impact on existing code.

\subsection{Weak References and Ephemerons}

Weak references introduce the notion of weak reachability in the heap. An object $\mathrm{x}$ is said to be weakly reachable if it is referred to by a weak reference. If $x$ is not also strongly reachable (through normal pointers), the garbage collector may collect $x$. The weak reference is said to be full if it points to a value, and empty if the value was erased by the GC.

An ephemeron [Bobot 2014; Hayes 1997] is an extension of a weak reference. It consists of a key and a value. The ephemeron weakly points to the key. The value is considered reachable if the ephemeron is reachable and the key is strongly reachable. Observe that ephemerons provide the mechanism to express conjunction of reachability relations, whereas the usual GC mechanism can only express disjunctions. If the key is not strongly reachable, the GC erases the reference to the key and the value. We call this process ephemeron sweeping. OCaml implements weak references as ephemerons with only the key but no value. Hence, we will only discuss ephemerons for the remainder of this section. Ephemerons in OCaml may have more than one key, and the behaviour is that, for the value to be considered reachable, the ephemeron and all of the keys are strongly reachable.

We would like the ephemeron marking and sweeping to also not require synchronizing all of the mutators and GC threads. To this end, each domain maintains a list of ephemerons it has created. This list is incrementally marked and swept by the owning domain concurrently with the execution on other domains. During domain termination, the ephemeron list is appended to a global ephemeron list which is adopted by one of the running domains.

5.1.1 Challenges. Ephemerons bring in several challenges for a concurrent GC. Since the reachability of ephemeron values can only be decided based on whether the keys are strongly reachable, ephemeron marking must be performed after all the domains have finished marking. During ephemeron marking, the domain local list of ephemerons is walked, and for each ephemeron, if the ephemeron and its key are strongly reachable, then the value is marked. Thus marking the ephemeron may introduce additional marking work. This complicates our termination algorithm (Section 3.2.1), as the number of domains with marking work no longer monotonically decreases. Moreover, the ephemeron value when marked may make other ephemerons reachable, which may be on the ephemeron list of another domain. Thus, if ephemeron marking introduces new mark work, we will need to go through another round of ephemeron marking.

OCaml ephemerons provide a get_key function which returns a strong reference to the key if the key is full (not erased by the GC). This strong reference is obtained by marking the key. If there were no other strong references to the key, then the get_key operation introduces additional mark work in the current cycle. Hence, just running the mutator may bring in additional mark work and cause a domain to need to mark even though it had previously finished the round's mark work. Only after all of the domains have marked their ephemerons and have not produced any new mark work can we proceed to the ephemeron sweep phase.

5.1.2 Ephemeron Marking. We extend the algorithm presented in Figure 3 to handle ephemeron marking (Figure 7). Due to the strict ordering requirements between ephemeron marking and sweeping, we introduce two phases in the GC - MARK and SWEEP_EPHE. If a program does not use ephemerons, then the SWEEP_EPHE phase can be skipped. The key idea is this: we say that 


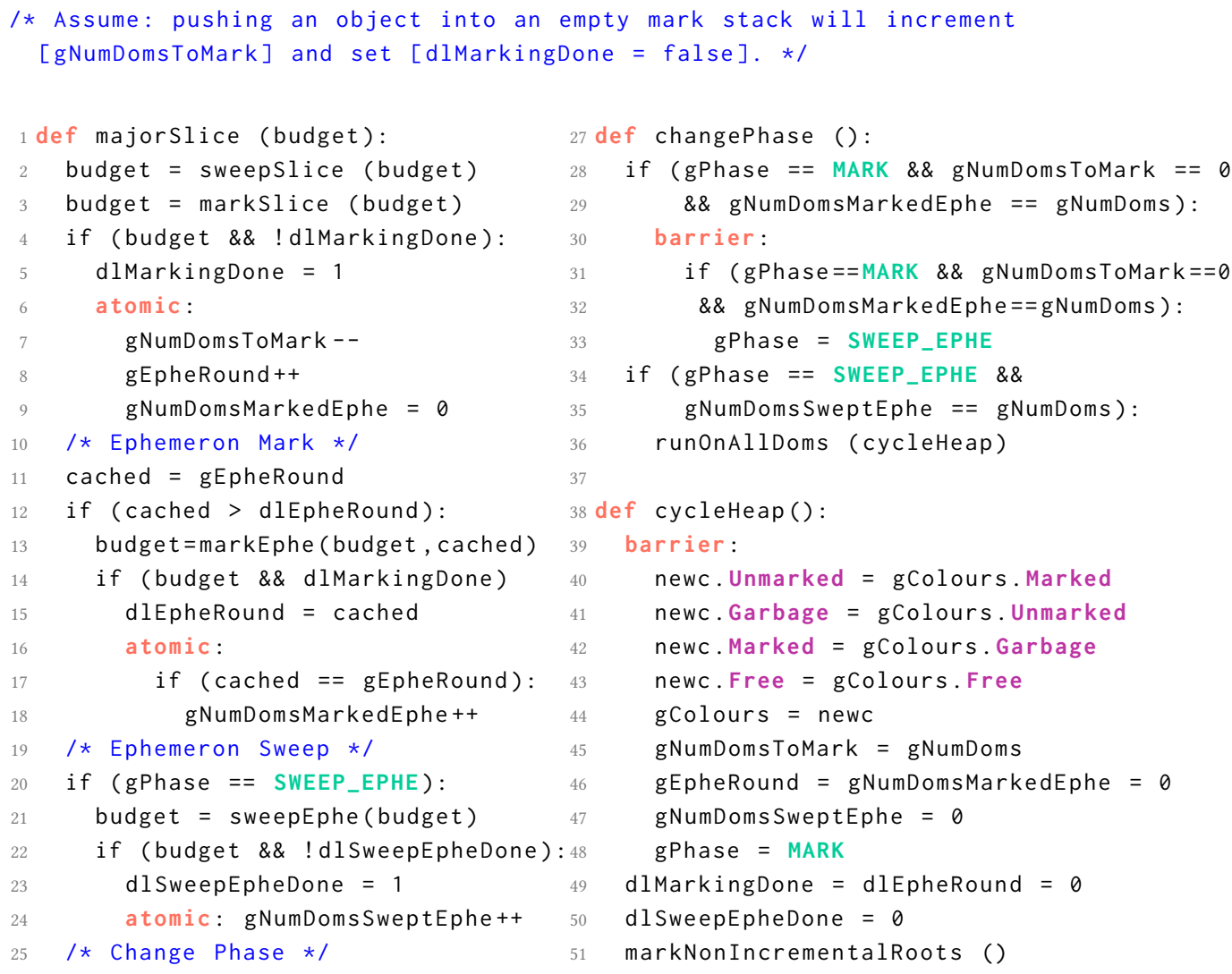

Fig. 7. Pseudocode for a slice of major heap work with ephemerons.

an ephemeron marking round is complete when a domain empties its mark stack. If all of the domains are able to mark the ephemerons in the same round and there is no new marking work generated, then the ephemeron marking phase is completed. Importantly, our solution does not require synchronization between each round of ephemeron marking.

For the sake of exposition, we assume that the number of domains (gNumDoms) remains constant. The global gNumDomsMarkedEphe tracks the number of domains that have marked their ephemerons in the current round. The global gNumDomsSweptEphe tracks the number of domains that have swept their ephemerons. The domain-local variable dlSweepEpheDone records whether the current domain has swept its ephemerons. We maintain the global ephemeron round in gEpheRound, and the last round for which a domain has marked its ephemerons in dlEpheRound. All of these variables are initialised to 0 at the start of the major cycle (see cycleHeap).

Whenever a domain empties its mark stack, it increments gEpheRound and sets gNumDomsMarkedEphe to 0 to indicate that no domains have marked ephemerons in the new round (lines 8-9). Before marking its ephemerons, a domain caches a copy of gEpheRound (line 11). If it completes marking and no new mark work has been generated, then it records that it has completed marking for the cached round (line 15). If another domain had not concurrently emptied its mark stack, then the cached round will be the same as the global round, and the domain increments the global count of domains that have completed marking in this current ephemeron round (lines 16-18). 
5.1.3 Termination. How do we know that marking is done? In each major cycle, gNumDomsMarkedEphe either increases from 0 to gNumDoms or is reset to 0 . Because of Ephemeron.get_key, gNumDomsMarkedEphe $==$ gNumDoms and gNumDomsToMark $==0$ can both be simultaneously true, however, running the mutator may create new mark work. This increments gNumDomsToMark, which will in turn necessitate an additional round of ephemeron marking. Hence, we check the termination condition again in a global barrier to verify that new mark work has not been created before switching to the SWEEP_EPHE phase (lines $28-33$ ). We have verified the implementation of the major slice work and termination in SPIN model checker [Holzmann 1997].

5.1.4 Ephemeron Sweeping. Sweeping ephemerons is comparatively simple. Each domain sweeps its ephemerons, clearing keys and values if necessary and increments gNumDomsSweptEphe. This variable monotonically increases to gNumDoms, at which point we cycle the major heap.

\subsection{Finalisers}

Each domain maintains a domain-local list of finalisers that it has installed, and handles finalization locally. OCaml has two variants of finalisation function:

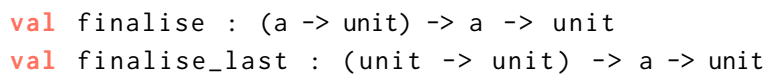

finalise applies the higher-order function on the object being finalised, and the function may revive the object by storing it in a global variable. Hence, as part of finalisation, the object is marked. Hence, these finalisers are marked to run when gNumDomsToMark first goes to 0 , but before any of the domains start marking ephemerons. Due to Ephemeron.get_key, we need a barrier to move from the main marking phase to marking finalisers.

The finalise_last finalisers do not get hold of the object being finalised. OCaml finalises them after all the marking has been completed for this major cycle i.e., after we enter SWEEP_EPHE phase. Similar to the other cases, we maintain a global variable each to keep track of the number of domains that have run their finalise and finalise_last finalisers. These variables are initialised to 0 and monotonically increase to gNumDoms. These variables are consulted before moving to the next corresponding GC phase. This mirrors the stock OCaml behaviour. The pseudocode for major slice with ephemerons and finalisers can be found in the Appendix A of the technical report [Sivaramakrishnan et al. 2020].

\subsection{Lazy Values}

OCaml has support for deferred computations through the use of lazy values, which interact with the GC. In stock OCaml, a lazy value ' a Lazy.t has one of the following representations:

- A block of size 1 with Lazy tag with a closure of type unit $\rightarrow$ ' $a$ in the first field.

- A block of size 1 with Forward tag with the computed value of type ' a in the first field.

- The computed value of type ' $a$ '.

When a lazy value with Lazy tag and closure $f$ is forced, the field is first updated to fun () $\rightarrow>$ raise Undefined, so that recursive forcing of a lazy value raises an exception. Then, the deferred computation $f$ is evaluated. If $f$ raises an exception e, then the computation is replaced with fun ()$\rightarrow>$ raise e. Otherwise, the mutator modifies the header to Forward and updates the first field to the result. Whenever the GC finds a reference to a Forward object, it may short-circuit the reference to directly point to the object.

This scheme is incompatible with a concurrent GC since the mutator modifying the word sized header may race with a GC thread which is marking the object, leading to one of the writes being

\footnotetext{
${ }^{1}$ We elide a subtlety with floats for the sake of exposition
} 
lost. Moreover, the writes to update the header to Forward and the field to the result of the deferred computation are non-atomic. Hence, one of the two domains concurrently forcing a lazy may witness one of the writes but not the other, leading to violation of type safety (segmentation fault). Moreover, given that the deferred computation may perform arbitrary side effects, it would be undesirable to duplicate the effects when two domains concurrently force a lazy value.

For Multicore OCaml, we have redesigned lazy values to make them safe for concurrent mutators and GC threads. We introduce a Forcing tag to indicate a lazy value currently being forced. When forcing a Lazy tagged lazy value, the tag is first updated to Forcing using a CAS. Then, the first field is updated to store the identifier of the current domain. Following this, the deferred computation is evaluated.

If the deferred computation encounters a lazy value with Forcing tag and the domain identifier in the first field matches the current domain identifier, then it indicates recursive forcing of this lazy value. If the domain identifiers are different, then it indicates concurrent forcing of this lazy by more than one domain. In both of these cases, we raise the Undefined exception.

If the computation results in a value, the first field is updated with the result, and the tag is now updated to Forward using a CAS. If the computation results in an exception, the first field is updated to a closure which when evaluated raises the same exception. Then, the tag is reset to Lazy using a CAS so that subsequent forcing of this lazy value raises the Undefined exception. The GC also marks Lazy and Forcing tagged objects with a CAS to handle the race between the mutator and the GC. Multicore-safe lazy still uses the same object layout as stock OCaml,

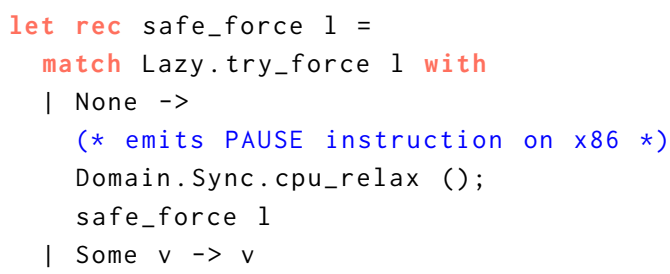

Fig. 8. Safely performing memoized computations in a concurrent setting but uses two CASes in the mutator and one CAS for GC marking each lazy value.

This design permits the common case of using lazy values for memoization in a concurrent setting. Given that we can distinguish between concurrent and recursive forcing of a lazy value, Multicore OCaml provides a Lazy.try_force: 'a Lazy.t $\rightarrow>$ 'a option primitive which behaves similar to Some (Lazy. force 1) except that it returns immediately with None if the lazy value 1 is already being forced concurrently by another domain. Using Lazy.try_force, one may safely access memoized computations by busy-waiting, for example, as shown in Figure 8.

\subsection{Fibers}

Multicore OCaml supports lightweight concurrency through language-level threads implemented using runtime support for heap-allocated, dynamically resized, stack segments (fibers) [Dolan et al. 2018b]. While the language support for concurrency is beyond the scope of the paper, care has to be taken towards the interaction between the concurrent GC and fibers. Multicore OCaml uses a deletion barrier which requires that the program stack be scanned at the start of the cycle. Since fibers are program stacks, before switching control to a fiber, all the objects on the fiber stack must be marked. This can lead to a race between a mutator wanting to switch to a fiber and other domains marking the fiber stack. Whenever a fiber stack is being scanned for marking, we obtain a lock on the fiber. If a mutator attempts to switch to a locked fiber, it spin waits until marking is complete. If a GC thread attempts to mark a locked fiber, it skips marking since the fiber is being marked. 


\section{EVALUATION}

In this section, we evaluate the performance of the two Multicore OCaml GC variants against the requirements listed in Section 1.3. We have implemented the multicore support by extending the OCaml 4.06.1 compiler.

The performance evaluation was performed on a 2-socket, Intel ${ }^{\circledR}$ Xeon ${ }^{\circledR}$ Gold 5120 x86-64 server, with 28 physical cores (14 cores on each socket), and 2 hardware threads per core. Each core runs at $2.20 \mathrm{GHz}$ and has $32 \mathrm{~KB}$ of L1 data cache, $32 \mathrm{~KB}$ of L1 instruction cache and $1 \mathrm{MB}$ of L2 cache. The cores on a socket share a 19.25 MB L3 cache. The server has 64GB of main memory and runs Ubuntu 18.04.

Since pause time measurements are sensitive to the operating system scheduling, we carefully set up the machine to eliminate measurement noise. We disable hardware threads, powersave and force a constant frequency of $2.20 \mathrm{GHz}$. We isolate 12 physical cores on each socket using Linux isolcpus for benchmarking. The kernel will not schedule any processes on these isolated cores unless explicitly requested. Thus, we have 12 cores each on 2 NUMA domains for benchmarking.

\subsection{Sequential Performance}

In this section, we analyse the performance of sequential OCaml programs on OCaml 4.06.1 (Stock), Multicore OCaml with the concurrent minor GC (ConcMinor) and the stop-the-world parallel minor GC (ParMinor). We ran Stock with default GC settings (next-fit collector, compaction is enabled).

Our aim is to determine whether unmodified sequential programs (modulo the changes required for the C API on the ConcMinor variant) running on the multicore variants retain throughput (running time) and latency (GC pause times). The sequential benchmark suite comprises of a mix of workloads including parsers (menhir, setrip, yojson), utilities (cpdf, decompress), option pricing (lexifi-g2pp), ray tracer (minilight), concurrency (*lwt*), bioinformatics (fasta, knucleotide, revcomp2, regexredux2), numerical analysis (matrix_multiplication, LU_decomposition), and simulation (game_of_life, nbody). The programs were run one after the other on one of the isolated cores with no other load on the machine.

6.1.1 Throughput. Figures 9a, 9b and 9c respectively show the normalized running time, the normalized maximum major heap size and the normalized major collection count of the sequential benchmarks on Multicore OCaml with Stock OCaml running time as the baseline.

On average (using geometric mean of the normalized running times), ConcMinor and ParMinor are $4.9 \%$ and $3.5 \%$ slower than Stock, respectively. ConcMinor is slower than ParMinor due to the overhead of the read barrier. This overhead itself manifests in two ways. First is the additional instructions that ConcMinor will have to execute over ParMinor. Secondly, since the read barrier is inlined at reads, the additional instructions tend to affect instruction cache efficiency. We have also observed that additional instructions due to read barrier in tight loops sometimes tend to disable or enable micro architectural optimisations, which tend to affect overall running time by a few percentage points. Hence, we believe that observed overheads are within reasonable bounds.

Focussing on specific benchmarks, pidigits 5 on Stock is $2.9 \times$ slower than the both of the multicore variants. This is due to the fact that pidigits 5 allocates $12.7 \mathrm{~GB}$ in the major heap in total, most of which are short-lived. This causes Stock to schedule 895 compactions and the benchmark spends $50 \%$ of its running time in the kernel allocating and freeing memory. By turning off compaction for pidigits, the runtime reduces to 2.21 seconds which is $5 \%(9 \%)$ faster than ConcMinor(ParMinor).

On the other side, game_of_life on multicore variants is $20 \%$ slower than Stock. game_of_life uses exceptions to encode the behaviour on out-of-bounds array access. Exceptions in the multicore variants are more expensive than Stock for two reasons. Firstly, the dedicated exception pointer 

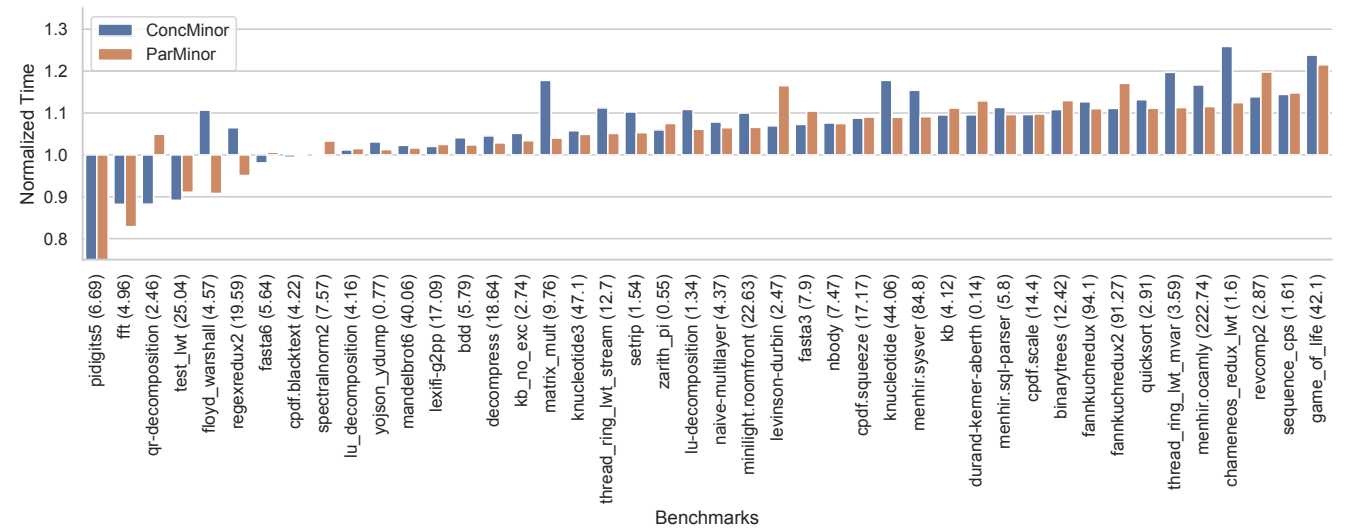

(a) Normalized runtime. Baseline is Stock OCaml whose running time in seconds is given in parenthesis.
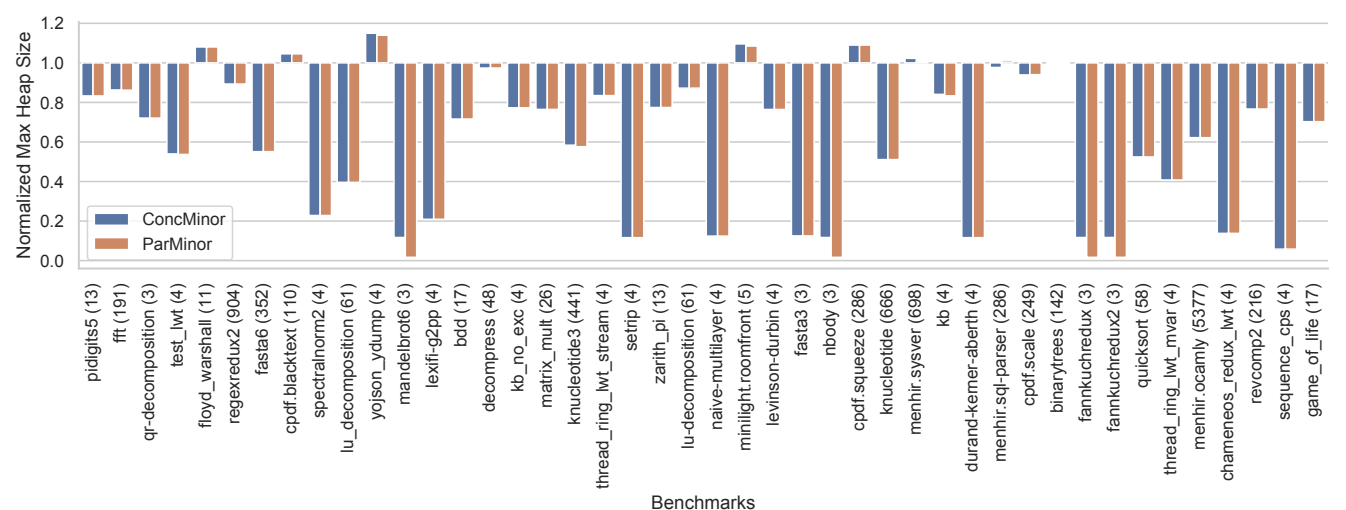

(b) Normalized maximum major heap size. Baseline is Stock OCaml whose maximum major heap size in MB is given in parenthesis.

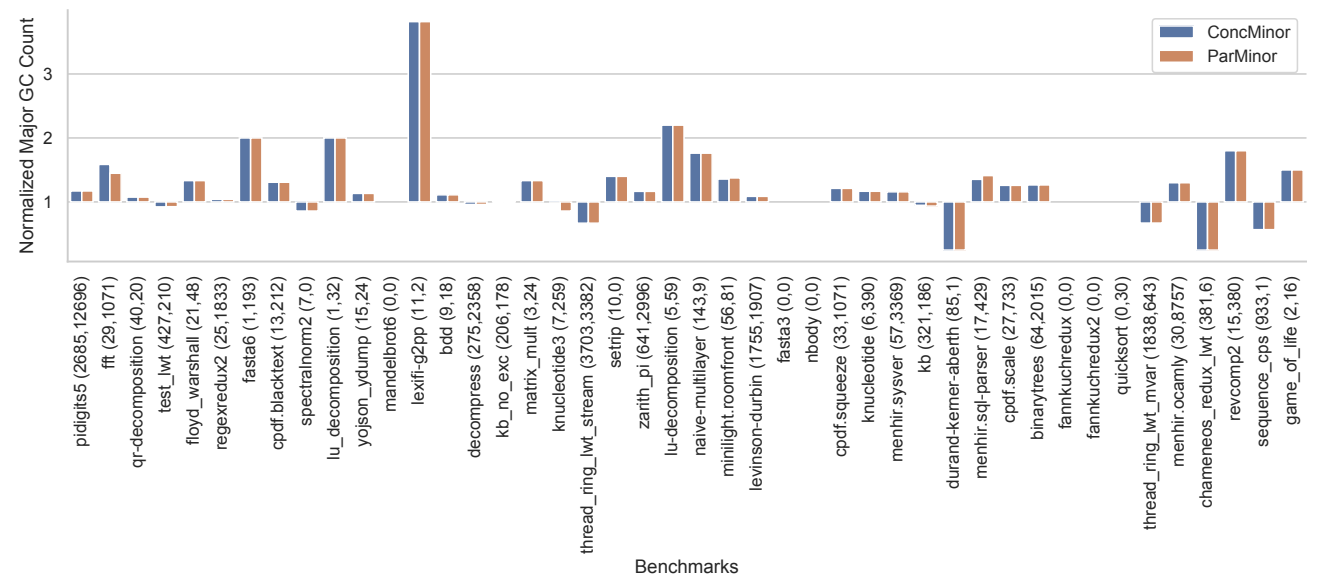

(c) Normalized major collection count. Baseline is Stock OCaml whose major collection count is given in first component in the parenthesis. The second component is the total allocation in the major heap in $\mathrm{MB}$ for Stock OCaml.

Fig. 9. Throughput of unmodified sequential OCaml programs on Multicore OCaml Proc. ACM Program. Lang., Vol. 4, No. ICFP, Article 113. Publication date: August 2020. 
register on x86-64 is now used for the pointer to the domain-local runtime structure, which holds the domain-local state including the exception pointer. Secondly, due to fibers in the multicore runtime, the stacks need to move to grow on stack overflow, unlike Stock. While Stock uses absolute addresses into the stack for exception pointers, we use relative addressing to encode the exception pointer to avoid rewriting the exception pointer when stacks are moved. Hence, the multicore variants need to execute additional instructions for setting up the exception handler and raising exceptions. We verified this by rewriting the benchmark using an optional type to capture the exception behaviour and the performance matches that of Stock. We experimented with the idea of retaining the dedicated exception pointer register in addition to the register for domain-local runtime state. In this case we observed performance that was generally worse; there was increased register pressure and consequently more spilling. Implementing absolute addressing for exceptions in the multicore variants would improve performance of exceptions, while making the (rarer) stack move operations slower.

On average, ConcMinor and ParMinor use 54\% and 61\% less memory than Stock (Figure 9b). Many of the benchmarks where the multicore variants do better than stock do not use much memory. However, multicore variants do better even on those benchmarks that allocates a lot of memory. For example, menhir. ocamly uses a maximum heap size of $5.3 \mathrm{~GB}$ on Stock and the multicore variants consume 3.3 GB. The next-fit collector that we use in Stock is susceptible to fragmentation whereas the size-segmented free list used in the multicore variants are tolerant to fragmentation. We ran the benchmark with first-fit collector and the benchmark did not terminate after 30 minutes. With the new best-fit collector which was added in OCaml 4.10.0, the benchmark runs in 250 seconds and consumes 3.3 GB, which matches the behaviour of multicore variants. The results show that the runtime and memory usage of sequential OCaml programs on the multicore variants is competitive with stock OCaml.

In our experiments, we use the same minor heap size (the OCaml default of $2 \mathrm{MB}$ ) for all the three variants. We observed that all the three variants allocate similar amount memory in the major heap. The average difference was less than $1 \%$. Hence, we report only the total allocations in the major heap for Stock (Figure 9c). We observe that the multicore variants tend to do a few more major collections than Stock. Although the variants allocate similar amounts of memory in the major heap, the allocators are different between Stock and the multicore variants, and vary in the amount of fragmentation in the heap. This induces differences in the GC major slice scheduling algorithm causing more major collections in the multicore variants.

6.1.2 Latency. Figure 10a and 10b shows the maximum and 99.9th percentile GC pause times of unmodified OCaml programs running on the different variants. We observe that both of the multicore variants remain competitive with Stock.

The most unresponsive benchmark is menhir.ocamly where the maximum pause times are 1125 ms, $689 \mathrm{~ms}$ and $635 \mathrm{~ms}$ on Stock, ParMinor (39\% lower than Stock) and ConcMinor (44\% lower then Stock). There are a number of benchmarks whose maximum pause times are over $10 \mathrm{~ms}$. We observed that in all of these programs, the maximum pause time is attributed to a large slice of major GC work.

While the GCs are incremental, the runtime computes a mark slice budget, the amount of incremental work (in memory words) that should be done in each cycle. The budget is computed based on the space overhead setting (percentage of memory used for live data), the current size of the major heap, the allocations since the last major slice, and a summarised history of work done in previous slices. In these benchmarks, there is a large amount of allocation since the last slice of major work, which causes the runtime to compute a very large budget, which in turn causes the large pause times. However, such huge imbalances are rare events. We can observe that the 


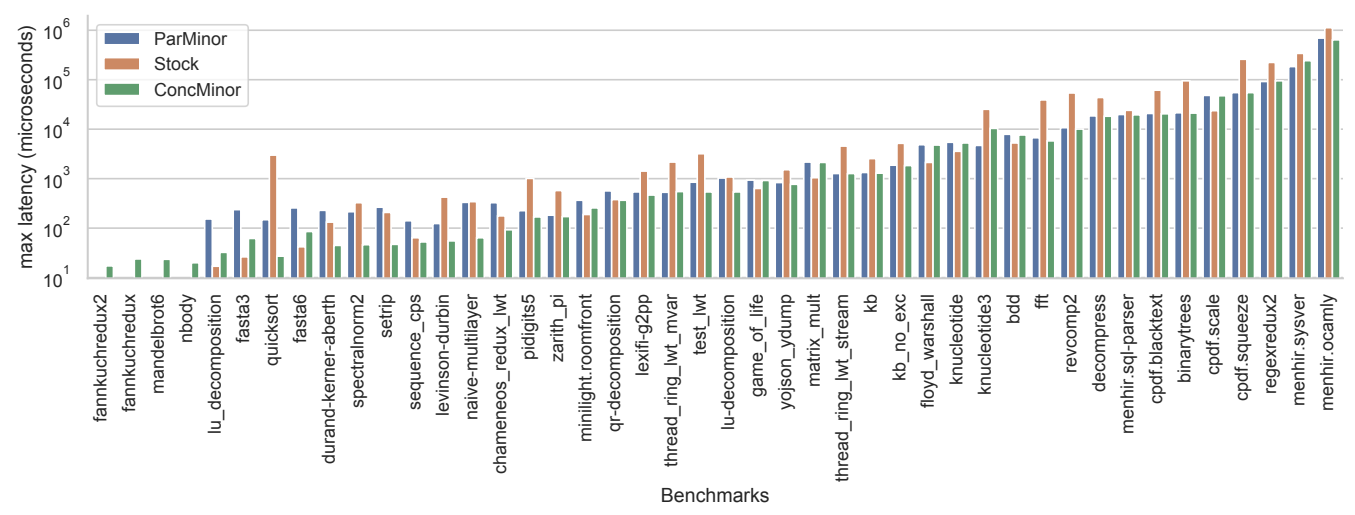

(a) Maximum GC pause times.

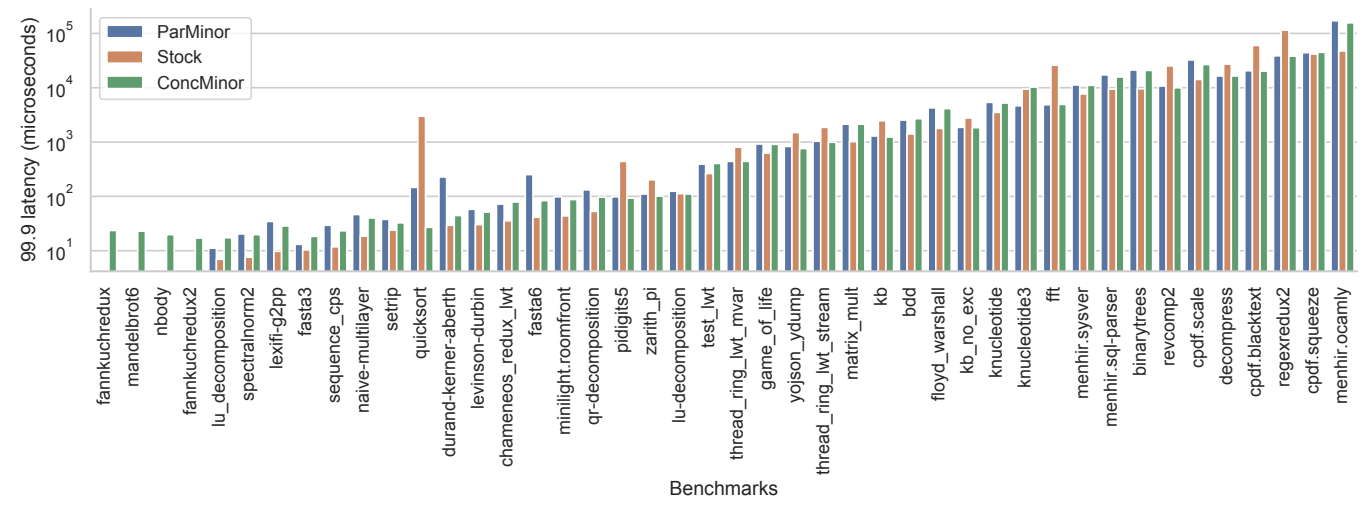

(b) 99.9 th percentile GC pause times.

Fig. 10. GC Pause times of unmodified sequential OCaml programs

99.9th percentile pause times are much better than the maximum pause times. For menhir. ocamly, 99.9th percentile pause times for Stock, ConcMinor and ParMinor are $47 \mathrm{~ms}, 155 \mathrm{~ms}$ and $171 \mathrm{~ms}$, respectively.

While these benchmarks are not interactive programs, the large pause times observed are antithetical to the common wisdom that OCaml is suitable for interactive programs. Are these large pause times indicative of the fundamental lack of responsiveness in the GC design or are they an artefact of the GC pacing algorithm? OCaml does not have an explicit setting for responsiveness; the space overhead parameter controls memory usage and only indirectly affects the pause times. We first observed that increasing the space overhead did not bring down the maximum pause time in menhir.ocamly.

We experimented with a threshold for the maximum budget for a mark slice. The threshold was chosen such that no major slice is longer than roughly $10 \mathrm{~ms}$ on the benchmarking machine. Without the maximum budget, menhir. ocamly on ConcMinor completed in $213 \mathrm{~s}$, with a maximum major heap size of $3.3 \mathrm{~GB}$ and a maximum latency of $635 \mathrm{~ms}$. With the maximum budget, the benchmark completed in $203 \mathrm{~s}$, with a maximum major heap size of $6.07 \mathrm{~GB}$ and a maximum latency of $10 \mathrm{~ms}$. While this approach will not ensure that the space overhead constraints are met, we are able to trade space for improvements in both the running time as well as maximum latency. This illustrates that our GC design does not fundamentally lack responsiveness. 

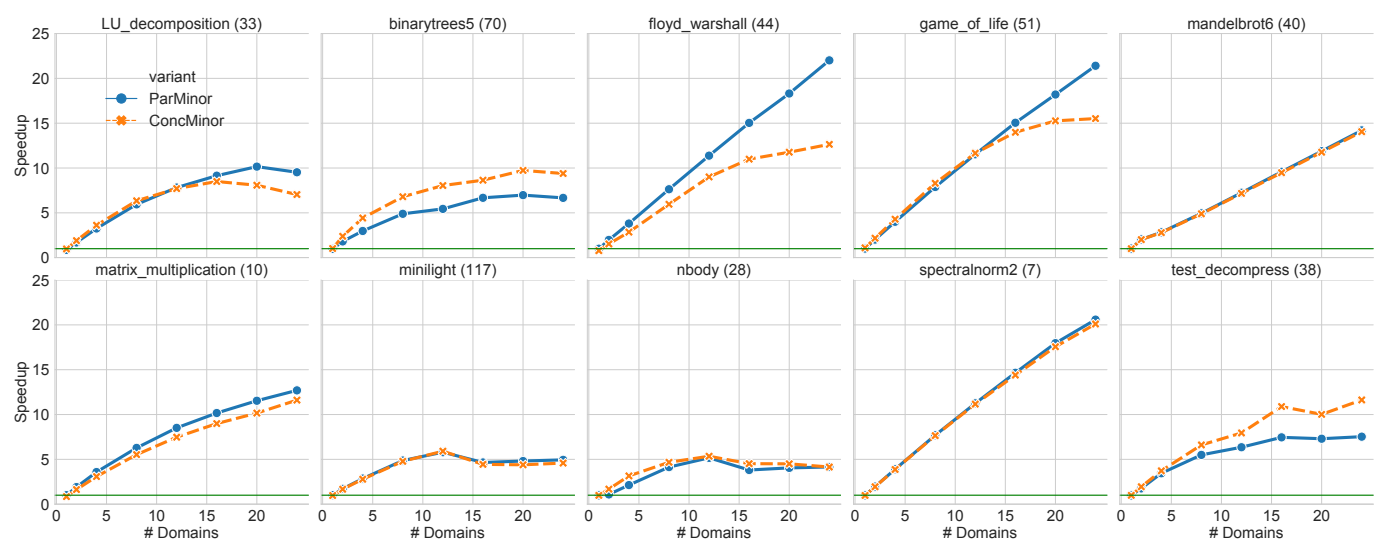

Fig. 11. Speedup of parallel benchmarks. The baseline running time in seconds is given in the parenthesis next to the benchmark name. The line parallel to the $x$-axis is at $y=1$.

\subsection{Parallel Benchmarks}

With parallel benchmarking, our aim is to determine whether the multicore variants scale with additional cores, and how the GC pause times are affected by additional core count. Our parallel benchmark suite is composed of numerical kernels (LU_decomposition, matrix_multiplication, spectralnorm2), ray tracing (minilight), GC stress test mirroring real workloads (binarytrees5), Zlib compression and decompression (test_decompress), simulations (game_of_life, nbody), allpairs shortest path algorithm (floyd_warshall) and Mandelbrot set plotting (mandelbrot6).

As mentioned earlier, we had isolated 12 cores each on the two sockets in the server. We ran each benchmark with the same input at different domain counts. For the domain counts less than or equal to 12, we pin them to the same socket using Linux taskset command. By default, Linux allocates pages in the same NUMA domain as the process generating the request. Hence, pinning the domains to the same NUMA domain minimises NUMA effects. For domain counts greater than 12 , they are allowed to span both sockets.

Figure 11 shows the speedup of the multicore variants. The baseline for speedup is the fastest sequential version of the program. The results show that ParMinor scales linearly for floyd_warshall, game_of_life and spectral_norm, whereas ConcMinor scales linearly only for spectral_norm. All of these benchmarks have a number of iterations with parallelisable work available in each iteration. In each iteration, the master domain farms out the work to the worker domains over a point to point channel data structure and synchronizes with the workers at the end.

In the case of ConcMinor, since the channels themselves are shared between the domains, they are in the major heap. When the master adds work in the channel, a pointer is created from the channel in the major heap to the work in the master domain's minor heap arena. When the worker fetches the work, it faults on the read and has to send an interrupt to the master domain to promote the work to the major heap. Until the master finishes promoting the work, the worker sits idle. This causes the gradual tapering out of speedup with increasing domain count. The master can eagerly promote the work, but this too has non-trivial overheads since at least the roots have to be scanned to ensure that there are no dangling references to the promoted object (Section 4.3.3). On the other hand, in the case of ParMinor, the worker directly reads the work item from master's minor heap arena. The other benchmarks have large non-parallelisable sections which causes sublinear speedup. Overall, when there is parallelisable work available, Multicore OCaml can effectively parallelise it. 
113:24 K.C. Sivaramakrishnan, S. Dolan, L. White, S. Jaffer, T. Kelly, A. Sahoo, S. Parimala, A. Dhiman, A. Madhavapeddy

Table 1. GC statistics for parallel benchmarks with 24 domains.

\begin{tabular}{|c|c|c|c|c|c|c|c|c|}
\hline \multirow{2}{*}{ Benchmark } & \multicolumn{2}{|c|}{ Major GCs } & \multicolumn{2}{|c|}{ Minor GCs } & \multicolumn{2}{|c|}{ Major Allocs (MB) } & \multicolumn{2}{|c|}{ Max Heap (MB) } \\
\hline & Conc & $\overline{\mathrm{Par}}$ & Conc & Par & Conc & Par & Conc & Par \\
\hline LU_decomposition & 98 & 15 & 66402 & 83469 & 141 & 134 & 100 & 101 \\
\hline binarytrees5 & 20 & 17 & 14084 & 17800 & 8088 & 7729 & 5124 & 7752 \\
\hline floyd_warshall & 15 & 15 & 409 & 424 & 19 & 13 & 13 & 13 \\
\hline game_of_life & 3 & 3 & 2 & 9 & 12 & 12 & 12 & 12 \\
\hline mandelbrot6 & 0 & 0 & 0 & 0 & 1 & 1 & 1 & 1 \\
\hline matrix_multiplication & 0 & 0 & 0 & 0 & 0 & 0 & 24 & 24 \\
\hline minilight & 44 & 6 & 116433 & 210146 & 272 & 249 & 48 & 166 \\
\hline nbody & 1 & 1 & 1 & 2 & 0 & 0 & 0 & 0 \\
\hline spectralnorm2 & 12 & 6 & 8249 & 10152 & 1 & 1 & 3 & 5 \\
\hline test_decompress & 16 & 4 & 22177 & 24218 & 5157 & 3693 & 2239 & 3851 \\
\hline
\end{tabular}

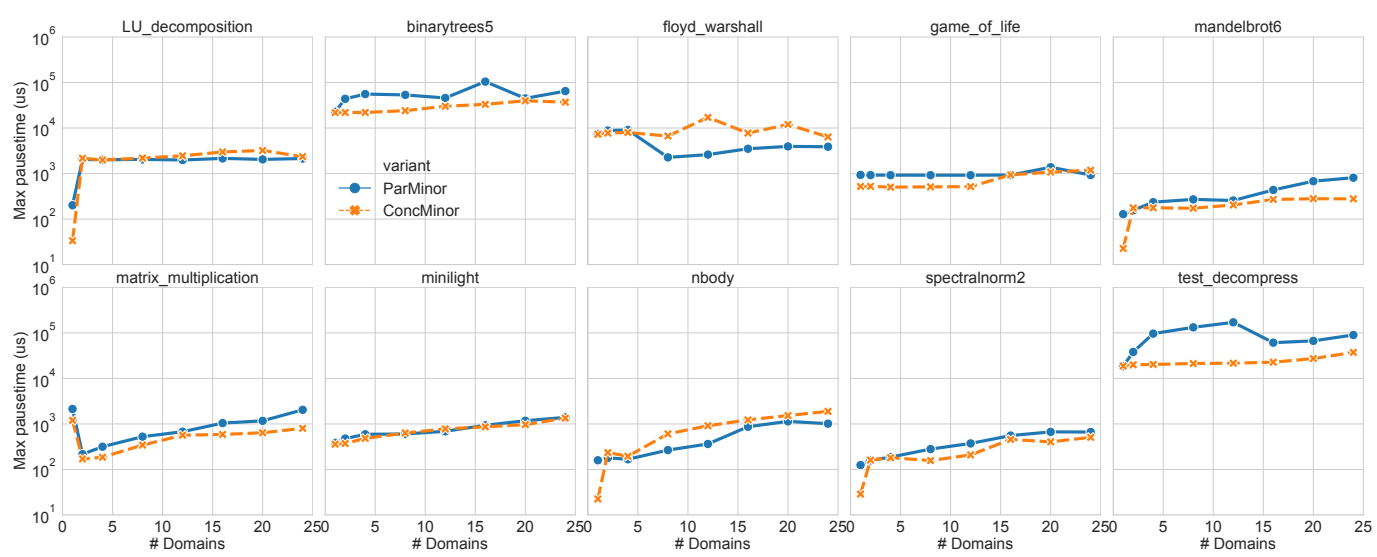

Fig. 12. Maximum GC pause times of parallel benchmarks.

Table 1 presents the GC statistics for the benchmark runs with 24 domains. We observe that ParMinor performs more minor GCs than ConcMinor. In the case of ParMinor whenever the heap arena of a domain fills up, the domain forces all the other domains to also collect their minor heaps. This issue can be fixed by having the domains request large pages for allocations from a contiguous minor heap space such that the minor collection is only performed when all of the minor heap is full. We also observe that ConcMinor allocate more in the major heap. This is due to the fact that any work that is shared between the domains need to be promoted to the major heap, whereas ParMinor can share without promotion.

Figure 12 shows the maximum GC pause times for the parallel benchmarks with increasing number of domains. We observe that the pause times of both the collectors are tolerant to increase in the number of domains. The maximum pause times remains the same or increases only marginally with increasing domain count. The worst case pause times for ParMinor is on test_decompress (170 $\mathrm{ms}$ at 12 domains) and ConcMinor is on binarytrees 5 ( $76 \mathrm{~ms}$ at 20 domains). For comparison, the maximum pause time for the sequential versions of the benchmarks on Stock OCaml are $43 \mathrm{~ms}$ for test_decompress and $94 \mathrm{~ms}$ for binarytrees5. Hence, the observed maximum latency numbers are within acceptable bounds. 
The maximum pause times were observed when a domain finishes a minor cycle and then runs a slice of the major heap work where it broadcasts a request for a stop-the-world barrier. An immediate improvement would be to separate out minor collection from the major slice work, as is the case in Stock, so that they are performed in two separate pauses. Hence, there is room for further improvements in pause times.

\section{DISCUSSION}

As we noted at the beginning of this paper, bringing parallelism to OCaml required us to find a point in the GC design space where sequential single core performance can be preserved but still introduce scalable parallelism as an option to OCaml developers. In this section, we will discuss the difficult design decisions that relate to this task of retrofitting parallelism onto an existing venerable functional language with a large existing codebase.

The core OCaml language had to be constrained in surprisingly few places where it exposed too much of the single-core internals. The obj module from the standard library is the biggest culprit; even though it is advertised as "not for the casual user", it is often used in the result of extracted code (e.g. from Coq) and the compiler itself. We primarily only had to remove two operations which update the header field of an object - Obj.set_tag and Obj.truncate. These operations conflict with concurrent GC threads non-atomically marking the object. Alternative primitives are provided that expose higher level functionality for some of the common use cases found.

Most of the changes elsewhere happened towards the parts of the language involving interfacing with the outside world. Signal handling, parts of the FFI, and marshalling required some modifications to make their semantics more robust in the face of parallelism, primarily by removing race conditions or shared state. In most cases, we simply had to tighten up the definition of allowable behaviour: for example, named callbacks to OCaml from $\mathrm{C}$ can no longer be mutated once registered using the raw pointer returned. We found no cases of this causing a problem in real world code.

The more advanced language features that had to be adapted for Multicore OCaml (ephemerons, lazy values and finalisers) are designed to minimise single-core performance impact. For example, lazy values introduce just two CAS operations into the value-forcing path, but none if already forced. Given that lazy values are introduced to memoize expensive computations the cost of the (almost always uncontended) CAS gets amortized. Multicore OCaml does not support compactions. We observed in our experiments that the size-segmented multicore allocator is more tolerant to fragmentation and performs as well as the best-fit collector added in OCaml 4.10.0 on menhir. ocamly. The result of this careful design is that OCaml users should be able to move gradually over to adopting multicore parallelism, without finding regressions (either semantically or performance-wise) in their existing large codebases.

When assessing the performance impact of our designs, we had to construct a micro- and macrobenchmark suite that is assembled from the wider OCaml community (primarily by extracting representative samples from the opam package manager). We immediately observed the difficulty of even small backwards compatibility changes between stock and multicore OCaml being difficult to adapt to in our own evaluations.

One primary culprit was the C API changes required by our original concurrent minor collector, which motivated us to build alternative designs to firmly determine the cost of adding read barriers. As we showed in our evaluation (§6), we found that our stop-the-world parallel minor collector that did not require changes to the OCaml FFI maintained extremely low pause times and good throughput. This happy result means that the multicore OCaml retrofit can be integrated into the mainstream OCaml distribution with very few user-observable compatibility concerns.

Multicore OCaml has also chosen to separate parallelism at the runtime level from concurrency at the language level. There is active research ongoing into various ways to integrate concurrency into 
OCaml via algebraic effects [Chandrasekaran et al. 2018; Dolan et al. 2018a]. From the perspective of the runtime, we have exposed just enough functionality via lightweight fibers to allow this research into strongly typed concurrent OCaml extensions to continue, but also not block the broader OCaml community from directly using the parallelism by spinning up domains using relatively low-level interfaces.

\section{RELATED WORK}

There are several notable works in the recent past that extend ML family languages with support for parallel programming. Manticore [Auhagen et al. 2011; Fluet et al. 2010] is a high-level parallel programming language with support for CML [Reppy 1993]-style coarse-grained parallelism as well as fine-grained parallelism in the form of parallel tuple expressions and parallel array comprehensions. The core language is a subset of Standard ML without mutable reference cells and arrays. MaPLe [Guatto et al. 2018; Raghunathan et al. 2016; Westrick et al. 2019] is a parallel extension of the MLton Standard ML compiler with support for nested parallelism. The programming model, as well as the garbage collector in MaPLe, takes advantage of disentaglement property which mandates that a thread cannot witness the memory allocations of another concurrent thread. Unlike these approaches, Multicore OCaml places no restriction on the programming model and sharing objects between domains, and aims to be fully compatible with the stock OCaml programming language.

SML\# features a concurrent, non-moving collector [Ueno and Ohori 2016] that is a similar approach to Multicore OCaml, but a single heap instead of a generational one. Multicore OCaml retains a generational approach in order to reduce the pressure on the major heap, at the cost of more complexity in the interaction between generations. SML\# allocator uses BiBoP [Steele Jr 1977] technique to distinguish used and free blocks in the free list to avoid adding a header to each object. Since OCaml objects already include a header, we reuse it in the free list and avoid having to use an auxiliary data structure. [Ueno and Ohori 2016] collector only supports a single GC thread. The paper also does not mention whether advanced data structures such as ephemerons, weak references and finalisers are supported. We observed that with multiple GC threads, careful attention has to be paid to the design of advanced data structures.

GHC has recently integrated an incremental, non-moving collector also inspired by the design in SML\# [Gamari and Dietz 2020]. GHC also maintains a nursery heap to allocate into rather than directly going into the major heap. Similar to SML\#, only one GC thread is currently supported with ongoing work to support multiple threads.

We retain the option to use the concurrent minor collector in a future revision of Multicore OCaml if it turns out to be necessary for many-core scalability. Although our stop-the-world minor collector scaled up admirably to at least 24 cores, synchronisation costs will inevitably increase as the number of cores do. Adding a read barrier (as used by the concurrent minor collector) opens up the GC design space considerably; for example to add a pauseless algorithm [Click et al. 2005] such as the ones used in modern Java GCs like Azul, ZGC [Tene et al. 2011] or Shenandoah [Flood et al. 2016]. Our results for the concurrent minor collector show that the read barrier has less overhead in OCaml code than initially expected, and would be a reasonable solution in many-core machines.

Several previous works [Anderson 2010; Auhagen et al. 2011; Doligez and Leroy 1993; Domani et al. 2002; Marlow and Peyton Jones 2011; Sivaramakrishnan et al. 2014] have explored concurrent minor collection with thread-private minor heap arenas. The [Marlow and Peyton Jones 2011] collector for GHC allows major to minor pointers and on-demand promotion, similarly to us. Our innovation here is the use of virtual memory mapping techniques in our concurrent minor collector (§4.3.2) to optimise the read barrier required to trap reads to a foreign minor heap arena.

Manticore [Auhagen et al. 2011] and MultiMLton [Sivaramakrishnan et al. 2014] use Appel-style semi-generational collection for the threads-local heaps. The local heap supports both minor and 
major collections. There is a dedicated shared heap in order to share objects between multiple threads. Similar to us Manticore permits pointers from the shared heap to the local heaps, and ondemand promotion. MultiMLton on the other hand does not permit pointers from the shared heap to the local heap, but takes advantage of ample user-level concurrency to preempts the execution of user-level threads that are about to introduce a shared to local heap pointer. In Manticore, the global heap is collector with a stop-the-world parallel collector, while MultiMLton uses a stop-the-world serial collector. In order to retain the low pause times of OCaml, Multicore OCaml uses concurrent mark-and-sweep collector for the major (shared) heap.

The Go programming language uses a concurrent, tri-colour, non-moving, mark-and-sweep, generational garbage collector and is designed for low GC pausetimes. Of particular interest is the interaction between goroutines and the GC. Before Multicore OCaml switches to an unmarked fiber, all the objects on the fiber stack are marked. This is necessary since Multicore OCaml uses a deletion barrier. Go opts for a different design where the goroutines are not marked before switching control to them. In turn, Go's write barrier is a combination of deletion and insertion barrier; until the current goroutine is shaded black, the write barrier marks both the object overwritten and the referrent [GoWriteBarrier 2015]. This design will be more responsive in concurrency heavy programs, at the cost of making write barriers more expensive even for code that does not use lightweight concurrency.

Our overall problem of retrofitting parallelism onto an existing sequential language has previously been explored by the Racket programming language as well. They added parallelism to a sequential runtime via two separate methods; firstly via a futures library that classifies operations that are parallel-safe as separate from ones that required more synchronisation [Swaine et al. 2010], and secondly via a places library that provides message-passing parallelism [Tew et al. 2011]. This approach is significantly less invasive to the Racket runtime than Multicore OCaml is to current mainline OCaml, but the parallel performance is reported as best suited to numerical tasks. Multicore OCaml aims to provide good parallel performance for algorithms involving large shared data structures, (such as those found in symbolic processing domains such as proof assistants), and also to integrate with existing debugging and tracing tools. Thus, we judged the significant engineering effort required to build the Multicore OCaml garbage collector as appropriate.

\section{CONCLUSIONS}

We present a novel, mostly-concurrent garbage collector that has been designed with the explicit goal of retrofitting parallelism onto OCaml while preserving performance and feature backwards compatibility. The extensive experimental evaluation validates that the new collector not only preserves backwards compatibility but also scale admirably on multiple cores.

All of the artefacts emerging from this research will be made available as open-source software and patches to the upstream OCaml distribution. The development repositories are available at https://github.com/ocaml-bench/sandmark for the benchmarking suite used in this paper, https: //github.com/ocaml-multicore/ocaml-multicore for the compiler forks, and https://github.com/ ocaml-multicore/multicore-ocaml-verify for the SPIN models.

\section{ACKNOWLEDGEMENTS}

We thank the anonymous reviewers, François Pottier, Pierre Chambart, Jon Harrop, Josh Berdine, Sam Goldman, and Guillaume Munch-Maccagnoni for their feedback on earlier drafts of the paper, Xavier Leroy and Damien Doligez and the core OCaml development team for their comments on the design of the Multicore OCaml GC. Portions of this research was funded via a Royal Commission for the Exhibition of 1851 and Darwin College Research Fellowships, and by grants from Jane Street and the Tezos Foundation. 


\section{REFERENCES}

Todd A. Anderson. 2010. Optimizations in a Private Nursery-Based Garbage Collector. In Proceedings of the 2010 International Symposium on Memory Management (Toronto, Ontario, Canada) (ISMM '10). Association for Computing Machinery, New York, NY, USA, 21-30. https://doi.org/10.1145/1806651.1806655

Andrew W Appel. 1990. A runtime system. Lisp and Symbolic Computation 3, 4 (1990), 343-380.

Sven Auhagen, Lars Bergstrom, Matthew Fluet, and John Reppy. 2011. Garbage Collection for Multicore NUMA Machines. In Proceedings of the 2011 ACM SIGPLAN Workshop on Memory Systems Performance and Correctness (San Jose, California) (MSPC '11). Association for Computing Machinery, New York, NY, USA, 51-57. https://doi.org/10.1145/1988915.1988929

François Bobot. 2014. Ephemerons meet OCaml GC. In OCaml Users and Developers Workshop.

Sivaramakrishnan Krishnamoorthy Chandrasekaran, Daan Leijen, Matija Pretnar, and Tom Schrijvers. 2018. Algebraic Effect Handlers go Mainstream (Dagstuhl Seminar 18172). Dagstuhl Reports 8, 4 (2018), 104-125. https://doi.org/10.4230/ DagRep.8.4.104

Cliff Click, Gil Tene, and Michael Wolf. 2005. The Pauseless GC Algorithm. In Proceedings of the 1st ACM/USENIX International Conference on Virtual Execution Environments (Chicago, IL, USA) (VEE '05). Association for Computing Machinery, New York, NY, USA, 46-56. https://doi.org/10.1145/1064979.1064988

Edsger W Dijkstra, Leslie Lamport, Alain J. Martin, Carel S. Scholten, and Elisabeth FM Steffens. 1978. On-the-fly garbage collection: An exercise in cooperation. Commun. ACM 21, 11 (1978), 966-975.

Stephen Dolan, Spiros Eliopoulos, Daniel Hillerström, Anil Madhavapeddy, KC Sivaramakrishnan, and Leo White. 2018a. Concurrent System Programming with Effect Handlers. In Trends in Functional Programming, Meng Wang and Scott Owens (Eds.). Springer International Publishing, Cham, 98-117.

Stephen Dolan, KC Sivaramakrishnan, and Anil Madhavapeddy. 2018b. Bounding Data Races in Space and Time. In Proceedings of the 39th ACM SIGPLAN Conference on Programming Language Design and Implementation (Philadelphia, PA, USA) (PLDI 2018). Association for Computing Machinery, New York, NY, USA, 242-255. https://doi.org/10.1145/ 3192366.3192421

Damien Doligez. 1989. Réalisation d'un glaneur de cellules de Lang et Dupont à générations. Memoire de DEA, Universite Paris VII (1989).

Damien Doligez. 2019. A new allocator for OCaml. http://gallium.inria.fr/ scherer/doc/chameau-sur-le-plateau/2019-10-08damien-doligez-major-allocator.org

Damien Doligez and Xavier Leroy. 1993. A Concurrent, Generational Garbage Collector for a Multithreaded Implementation of ML. In Proceedings of the 20th ACM SIGPLAN-SIGACT Symposium on Principles of Programming Languages (Charleston, South Carolina, USA) (POPL '93). Association for Computing Machinery, New York, NY, USA, 113-123. https://doi.org/ $10.1145 / 158511.158611$

Tamar Domani, Gal Goldshtein, Elliot K. Kolodner, Ethan Lewis, Erez Petrank, and Dafna Sheinwald. 2002. Thread-Local Heaps for Java. In Proceedings of the 3rd International Symposium on Memory Management (Berlin, Germany) (ISMM '02). Association for Computing Machinery, New York, NY, USA, 76-87. https://doi.org/10.1145/512429.512439

Marc Feeley. 1993. Polling Efficiently on Stock Hardware. In Proceedings of the Conference on Functional Programming Languages and Computer Architecture (Copenhagen, Denmark) (FPCA '93). Association for Computing Machinery, New York, NY, USA, 179-187. https://doi.org/10.1145/165180.165205

Christine H. Flood, Roman Kennke, Andrew Dinn, Andrew Haley, and Roland Westrelin. 2016. Shenandoah: An Open-Source Concurrent Compacting Garbage Collector for OpenJDK. In Proceedings of the 13th International Conference on Principles and Practices of Programming on the fava Platform: Virtual Machines, Languages, and Tools (Lugano, Switzerland) (PPPf'16). Association for Computing Machinery, New York, NY, USA, Article 13, 9 pages. https://doi.org/10.1145/2972206.2972210

Matthew Fluet, Mike Rainey, John Reppy, and Adam Shaw. 2010. Implicitly Threaded Parallelism in Manticore. F. Funct. Program. 20, 5-6 (Nov. 2010), 537-576. https://doi.org/10.1017/S0956796810000201

Ben Gamari and Laura Dietz. 2020. Alligator Collector: A Latency-Optimized Garbage Collector for Functional Programming Languages. In Proceedings of the 2020 ACM SIGPLAN International Symposium on Memory Management (London, UK) (ISMM 2020). Association for Computing Machinery, New York, NY, USA, 87âĂŞ99. https://doi.org/10.1145/3381898. 3397214

Peter Gammie, Antony L. Hosking, and Kai Engelhardt. 2015. Relaxing Safely: Verified on-the-Fly Garbage Collection for X86-TSO. In Proceedings of the 36th ACM SIGPLAN Conference on Programming Language Design and Implementation (Portland, OR, USA) (PLDI '15). Association for Computing Machinery, New York, NY, USA, 99-109. https://doi.org/10. $1145 / 2737924.2738006$

GoWriteBarrier 2015. Go Write Barrier. https://golang.org/src/runtime/mbarrier.go

David Gries. 1977. An Exercise in Proving Parallel Programs Correct. Commun. ACM 20, 12 (Dec. 1977), 921-930. https://doi.org/10.1145/359897.359903

Adrien Guatto, Sam Westrick, Ram Raghunathan, Umut Acar, and Matthew Fluet. 2018. Hierarchical Memory Management for Mutable State. In Proceedings of the 23rd ACM SIGPLAN Symposium on Principles and Practice of Parallel Programming

Proc. ACM Program. Lang., Vol. 4, No. ICFP, Article 113. Publication date: August 2020. 
(Vienna, Austria) (PPoPP '18). Association for Computing Machinery, New York, NY, USA, 81-93. https://doi.org/10. $1145 / 3178487.3178494$

Barry Hayes. 1997. Ephemerons: A New Finalization Mechanism. In Proceedings of the 12th ACM SIGPLAN Conference on Object-Oriented Programming, Systems, Languages, and Applications (Atlanta, Georgia, USA) (OOPSLA '97). Association for Computing Machinery, New York, NY, USA, 176-183. https://doi.org/10.1145/263698.263733

Gerard J. Holzmann. 1997. The Model Checker SPIN. IEEE Trans. Softw. Eng. 23, 5 (May 1997), 279-295. https://doi.org/10. $1109 / 32.588521$

Lorenz Huelsbergen and Phil Winterbottom. 1998. Very Concurrent Mark-\&-Sweep Garbage Collection without Fine-Grain Synchronization. In Proceedings of the 1st International Symposium on Memory Management (Vancouver, British Columbia, Canada) (ISMM '98). Association for Computing Machinery, New York, NY, USA, 166-175. https://doi.org/10.1145/ 286860.286878

Richard Jones, Antony Hosking, and Eliot Moss. 2011. The Garbage Collection Handbook: The Art of Automatic Memory Management (1st ed.). Chapman \& Hall/CRC.

Xavier Leroy. 1990. The ZINC experiment : an economical implementation of the ML language. Technical Report RT-0117. INRIA. 100 pages. https://hal.inria.fr/inria-00070049

Anil Madhavapeddy, Richard Mortier, Charalampos Rotsos, David Scott, Balraj Singh, Thomas Gazagnaire, Steven Smith, Steven Hand, and Jon Crowcroft. 2013. Unikernels: Library Operating Systems for the Cloud. In Proceedings of the Eighteenth International Conference on Architectural Support for Programming Languages and Operating Systems (Houston, Texas, USA) (ASPLOS '13). Association for Computing Machinery, New York, NY, USA, 461-472. https://doi.org/10.1145/ 2451116.2451167

Simon Marlow and Simon Peyton Jones. 2011. Multicore Garbage Collection with Local Heaps. In Proceedings of the International Symposium on Memory Management (San Jose, California, USA) (ISMM '11). Association for Computing Machinery, New York, NY, USA, 21-32. https://doi.org/10.1145/1993478.1993482

Simon Marlow, Simon Peyton Jones, and Satnam Singh. 2009. Runtime Support for Multicore Haskell. In Proceedings of the 14th ACM SIGPLAN International Conference on Functional Programming (Edinburgh, Scotland) (ICFP '09). Association for Computing Machinery, New York, NY, USA, 65-78. https://doi.org/10.1145/1596550.1596563

Yaron Minsky, Anil Madhavapeddy, and Jason Hickey. 2013. Real World OCaml - Functional Programming for the Masses O’Reilly. http://shop.oreilly.com/product/0636920024743.do\#tab_04_2

OCamlCAPI 2019. Interfacing C with OCaml. https://caml.inria.fr/pub/docs/manual-ocaml/intfc.html

Ram Raghunathan, Stefan K. Muller, Umut A. Acar, and Guy Blelloch. 2016. Hierarchical Memory Management for Parallel Programs. In Proceedings of the 21st ACM SIGPLAN International Conference on Functional Programming (Nara, Japan) (ICFP 2016). Association for Computing Machinery, New York, NY, USA, 392-406. https://doi.org/10.1145/2951913.2951935

ReasonML 2019. ReasonML : A new syntax and toolchain for OCaml. https://reasonml.github.io/

John H. Reppy. 1993. Concurrent ML: Design, Application and Semantics. In Functional Programming, Concurrency, Simulation and Automated Reasoning: International Lecture Series 1991-1992, McMaster University, Hamilton, Ontario, Canada. Springer-Verlag, Berlin, Heidelberg, 165-198.

Scott Schneider, Christos D. Antonopoulos, and Dimitrios S. Nikolopoulos. 2006. Scalable Locality-Conscious Multithreaded Memory Allocation. In Proceedings of the 5th International Symposium on Memory Management (Ottawa, Ontario, Canada) (ISMM '06). Association for Computing Machinery, New York, NY, USA, 84-94. https://doi.org/10.1145/1133956.1133968

KC Sivaramakrishnan, Stephen Dolan, Leo White, Sadiq Jaffer, Tom Kelly, Anmol Sahoo, Sudha Parimala, Atul Dhiman, and Anil Madhavapeddy. 2020. Retrofitting Parallelism onto OCaml. ArXiv abs/2004.11663 (2020). https://arxiv.org/abs/2004 11663

KC Sivaramakrishnan, Lukasz Ziarek, and Suresh Jagannathan. 2012. Eliminating Read Barriers through Procrastination and Cleanliness. In Proceedings of the 2012 International Symposium on Memory Management (Beijing, China) (ISMM '12). Association for Computing Machinery, New York, NY, USA, 49-60. https://doi.org/10.1145/2258996.2259005

KC Sivaramakrishnan, Lukasz Ziarek, and Suresh Jagannathan. 2014. MultiMLton: A multicore-aware runtime for standard ML. J. Funct. Program. 24, 6 (2014), 613-674. https://doi.org/10.1017/S0956796814000161

Daniel Dominic Sleator and Robert Endre Tarjan. 1985. Self-Adjusting Binary Search Trees. F. ACM 32, 3 (July 1985), 652-686. https://doi.org/10.1145/3828.3835

Thomas A. Standish. 1980. Data Structure Techniques. Addison-Wesley Longman Publishing Co., Inc., USA.

Guy Lewis Steele Jr. 1977. Data Representations in PDP-10 MacLISP. Technical Report. Massauchusetts Institute of Technology Cambridge Artificial Intelligence Laboratory.

James Swaine, Kevin Tew, Peter Dinda, Robert Bruce Findler, and Matthew Flatt. 2010. Back to the Futures: Incremental Parallelization of Existing Sequential Runtime Systems. In Proceedings of the ACM International Conference on Object Oriented Programming Systems Languages and Applications (Reno/Tahoe, Nevada, USA) (OOPSLA '10). Association for Computing Machinery, New York, NY, USA, 583-597. https://doi.org/10.1145/1869459.1869507 
113:30 K.C. Sivaramakrishnan, S. Dolan, L. White, S. Jaffer, T. Kelly, A. Sahoo, S. Parimala, A. Dhiman, A. Madhavapeddy

Gil Tene, Balaji Iyengar, and Michael Wolf. 2011. C4: The Continuously Concurrent Compacting Collector. SIGPLAN Not. 46, 11 (June 2011), 79-88. https://doi.org/10.1145/2076022.1993491

Kevin Tew, James Swaine, Matthew Flatt, Robert Bruce Findler, and Peter Dinda pdinda@northwestern.edu. 2011. Places: Adding Message-Passing Parallelism to Racket. In Proceedings of the 7th Symposium on Dynamic Languages (Portland, Oregon, USA) (DLS '11). Association for Computing Machinery, New York, NY, USA, 85-96. https://doi.org/10.1145/ 2047849.2047860

Katsuhiro Ueno and Atsushi Ohori. 2016. A Fully Concurrent Garbage Collector for Functional Programs on Multicore Processors. SIGPLAN Not. 51, 9 (Sept. 2016), 421-433. https://doi.org/10.1145/3022670.2951944

Martin T Vechev, David F Bacon, Perry Cheng, and David Grove. 2005. Derivation and evaluation of concurrent collectors. In European Conference on Object-Oriented Programming. Springer, 577-601.

Sam Westrick, Rohan Yadav, Matthew Fluet, and Umut A. Acar. 2019. Disentanglement in Nested-Parallel Programs. Proc. ACM Program. Lang. 4, POPL, Article 47 (Dec. 2019), 32 pages. https://doi.org/10.1145/3371115

T. Yuasa. 1990. Real-Time Garbage Collection on General-Purpose Machines. F. Syst. Softw. 11, 3 (March 1990), 181-198. https://doi.org/10.1016/0164-1212(90)90084-Y 